\title{
An experimental and theoretical study of the photoelectron spectra of cis-dichloroethene: Valence shell vertical ionization and vibronic coupling in the low-lying cationic states
}

\author{
A. B. Trofimov, ${ }^{1,2}$ I. Powis,${ }^{3}$ R. C. Menzies, ${ }^{3}$ D. M. P. Holland,${ }^{4}$ E. Antonsson, ${ }^{5}$ \\ M. Patanen, ${ }^{6}$ C. Nicolas, ${ }^{7}$ C. Miron, ${ }^{8,9}$ A. D. Skitnevskaya, ${ }^{1}$ E. V. Gromov, ${ }^{10}$ and \\ H. Köppel ${ }^{10}$ \\ ${ }^{1}$ Laboratory of Quantum Chemistry, Irkutsk State University, Karl Marx Str. 1, 664003 \\ Irkutsk, Russia \\ ${ }^{2}$ Favorsky's Institute of Chemistry, SB RAS, Favorsky Str. 1, 664033 Irkutsk, Russia \\ ${ }^{3}$ School of Chemistry, University of Nottingham, Nottingham NG7 2RD, United Kingdom \\ ${ }^{4}$ Daresbury Laboratory, Daresbury, Warrington, Cheshire WA4 4AD, United Kingdom \\ ${ }^{5}$ Physical Chemistry, Freie Universität Berlin, Takustr. 3, D-14195 Berlin, Germany \\ ${ }^{6}$ Nano and Molecular Systems Research Unit, Faculty of Science, P. O. Box 3000, 90014 \\ University of Oulu, Finland \\ ${ }^{7}$ Synchrotron SOLEIL, l'Orme des Merisiers, Saint-Aubin, BP 48, 91192 Gif-sur-Yvette, \\ France \\ ${ }^{8}$ LIDYL, CEA, CNRS, Université Paris-Saclay, CEA Saclay, 91191 Gif-sur-Yvette, France \\ ${ }^{9}$ Extreme Light Infrastructure-Nuclear Physics (ELI-NP), "Horia Hulubei" National Institute \\ for Physics and Nuclear Engineering, 077125, Măgurele, Jud. Ilfov, Romania \\ ${ }^{10}$ Theoretische Chemie, Physikalisch-Chemisches Institut, Universität Heidelberg, Im \\ Neuenheimer Feld 229, 69120 Heidelberg, Germany
}

\begin{abstract}
The valence shell photoelectron spectrum of cis-dichloroethene has been studied both experimentally and theoretically. Photoelectron spectra have been recorded with horizontally and vertically plane polarized synchrotron radiation, thereby allowing the anisotropy parameters, characterizing the angular distributions, to be determined. The third-order algebraic-diagrammatic construction $(\mathrm{ADC}(3))$ approximation scheme for the one-particle Green's function has been employed to compute the complete valence shell ionization spectrum. In addition, the vertical ionization energies have been calculated using the outer valence Green's function method (OVGF) and the equation-of-motion (EOM) coupled cluster (CC) theory at the level of the EOM-IP-CCSD model. The theoretical results have enabled assignments to be proposed for most of the structure observed in the experimental spectra,
\end{abstract}


including the inner-valence regions dominated by satellite states. The linear vibronic coupling model has been employed to study the vibrational structure of the lowest photoelectron bands, using parameters obtained from $a b$ initio calculations. The ground state optimized geometries and vibrational frequencies have been computed at the level of the second-order MøllerPlesset perturbation theory, and the dependence of the ionization energies on the nuclear configuration has been evaluated using the OVGF method. While the adiabatic approximation holds for the $\tilde{\mathrm{X}}^{2} \mathrm{~B}_{1}$ state photoelectron band, the $\tilde{\mathrm{A}}^{2} \mathrm{~B}_{2}, \tilde{\mathrm{B}}^{2} \mathrm{~A}_{1}$, and $\tilde{\mathrm{C}}^{2} \mathrm{~A}_{2}$ states interact vibronically and form a complex photoelectron band system with four distinct maxima. The $\tilde{\mathrm{D}}^{2} \mathrm{~B}_{1}$ and $\tilde{\mathrm{E}}^{2} \mathrm{~B}_{2}$ states also interact vibronically with each other. The potential energy surface of the $\tilde{\mathrm{D}}^{2} \mathrm{~B}_{1}$ state is predicted to have a double-minimum shape with respect to the out-ofplane $a_{2}$ deformations of the molecular structure. The single photoelectron band resulting from this interaction is characterized by a highly irregular structure, reflecting the nonadiabatic nuclear dynamics occurring on the two coupled potential energy surfaces forming a conical intersection close to the minimum of the $\tilde{\mathrm{E}}^{2} \mathrm{~B}_{2}$ state. 


\section{INTRODUCTION}

The Born-Oppenheimer approximation, ${ }^{1}$ which separates the electronic and nuclear motions, allows molecular processes to be described as being due to nuclei moving over the potential energy surfaces formed by the electrons. Each electronic state is characterized by its own potential energy surface which is decoupled from those of other electronic states. This picture of non-interacting states has proved highly successful in interpreting the photoelectron spectra of numerous molecules. ${ }^{2-4}$ Under these conditions, the regular vibrational progressions associated with a specific photoelectron band can be simulated by using the Franck-Condon factors connecting the initial neutral and the final ionic states. ${ }^{5}$ However, experiments have shown that in many molecules the vibrational structure becomes erratic, resulting in diffuse bands exhibiting no regular progressions. This irregular structure can be attributed to a breakdown of the Born-Oppenheimer approximation, and is often observed in polyatomic molecules where there is a large number of energetically close-lying electronic states and many nuclear degrees of freedom. ${ }^{6-12}$

Non-adiabatic effects, due to the coupling between electronic and nuclear motions, can lead to the formation of a conical intersection between potential energy surfaces. Such vibronic coupling, by providing a highly efficient pathway between neighbouring electronic states, can lead to photoelectron bands displaying complex vibrational excitations involving not only the totally symmetric modes but also the non-symmetric modes which are normally forbidden (at least in odd quanta). The development of the theoretical aspects of vibronic coupling has been summarized in several reviews. ${ }^{6,7,9,10}$ These show that vibronic coupling complicates ionization spectra and can result in photoelectron bands exhibiting irregular vibrational structure and also in the appearance of unexpected bands. ${ }^{13}$

The present work concerns the photoelectron spectrum of cis-dichloroethene $\left(\mathrm{C}_{2} \mathrm{Cl}_{2} \mathrm{H}_{2}\right.$, Figure 1), henceforth referred to simply as dichloroethene. Synchrotron radiation has been employed to record valence shell photoelectron spectra in the photon excitation range $19-90$ $\mathrm{eV}$. The lowest energy band, assigned to the $\left(3 \mathrm{~b}_{1}\right)^{-1} \tilde{\mathrm{X}}^{2} \mathrm{~B}_{1}$ state, exhibits prolonged vibrational progressions which can be analyzed in terms of excitations involving the totally symmetric 
modes. In contrast, in the bands associated with the $\left(9 \mathrm{~b}_{2}\right)^{-1} \tilde{\mathrm{A}}^{2} \mathrm{~B}_{2},\left(10 \mathrm{a}_{1}\right)^{-1} \tilde{\mathrm{B}}^{2} \mathrm{~A}_{1}$ and $\left(2 \mathrm{a}_{2}\right)^{-}$ ${ }^{1} \tilde{\mathrm{C}}^{2} \mathrm{~A}_{2}$ states, appearing in the binding energy range $\sim 11.2-13.1 \mathrm{eV}$, regular vibrational structure is only observed in the low energy portion of the $\tilde{\mathrm{A}}^{2} \mathrm{~B}_{2}$ state band. The remaining features associated with these states are rather diffuse and structureless. The most surprising issue for the band system comprising the $\tilde{\mathrm{A}}^{2} \mathrm{~B}_{2}, \tilde{\mathrm{B}}^{2} \mathrm{~A}_{1}$ and $\tilde{\mathrm{C}}^{2} \mathrm{~A}_{2}$ states is that the experimental spectrum contains four distinct maxima with three contributing cationic states. The next two states, $\left(2 \mathrm{~b}_{1}\right)^{-1} \tilde{\mathrm{D}}^{2} \mathrm{~B}_{1}$ and $\left(8 \mathrm{~b}_{2}\right)^{-1} \tilde{\mathrm{E}}^{2} \mathrm{~B}_{2}$ form a single photoelectron band with highly irregular vibrational structure.

The unusual photoelectron band shapes observed in the experimental spectra of dichloroethene arise from vibronic coupling and the breakdown of the adiabatic approximation. Such effects can be expected for two groups of cationic states, namely the $\left(\tilde{\mathrm{A}}^{2} \mathrm{~B}_{2}, \tilde{\mathrm{B}}^{2} \mathrm{~A}_{1}\right.$, and $\left.\tilde{\mathrm{C}}^{2} \mathrm{~A}_{2}\right)$ and the $\left(\tilde{\mathrm{D}}^{2} \mathrm{~B}_{1}\right.$ and $\left.\tilde{\mathrm{E}}^{2} \mathrm{~B}_{2}\right)$ states, due to the relatively small vertical energy gaps between the states within each group. In the first group, the states can be coupled via the $b_{1}, a_{2}$ and $b_{2}$ non-totally symmetric modes $\left(\mathrm{B}_{2} \times \mathrm{A}_{2} \times b_{1} \supset \mathrm{A}_{1}, \mathrm{~A}_{2} \times \mathrm{A}_{1} \times a_{2} \supset \mathrm{A}_{1}\right.$, $\left.\mathrm{B}_{2} \times \mathrm{A}_{1} \times b_{2} \supset \mathrm{A}_{1}\right)$, and in the second group via the $a_{2}$ modes $\left(\mathrm{B}_{1} \times \mathrm{B}_{2} \times a_{2} \supset \mathrm{A}_{1}\right)$.

We have investigated the vibronic coupling effects outlined above by employing the theoretical approach which has been applied previously to various vibronic coupling systems. ${ }^{6,14}$ In this approach, the nuclear dynamics are described using model Hamiltonians assuming linear vibronic coupling (LVC) between the electronic states, expressed in the socalled diabatic basis. ${ }^{6,14}$ The parameters required for the LVC models are derived from $a b$ initio calculations using the outer valence Green's function (OVGF) method ${ }^{15-17}$ and the second-order Møller-Plesset perturbation theory (MP2). The results obtained from our vibronic coupling calculations provide a satisfactory explanation for most of the structure associated with the outer valence photoelectron bands.

In addition to affecting the electronic structure, previous theoretical ${ }^{18,19}$ and experimental $^{20}$ studies have established that vibronic coupling also influences the photoionization dynamics. We have investigated the effect of such interactions by measuring photoelectron angular distributions for all the outer valence shell electronic states of 
dichloroethene. The results show that the angular distributions associated with a particular electronic state are modified by the neighbouring state to which it is coupled.

Of relevance to the present work are photoelectron spectra of dichloroethene recorded with $\mathrm{HeI},{ }^{21-25} \mathrm{HeII},{ }^{25} \mathrm{Al} \mathrm{K \alpha},{ }^{26}$ and synchrotron, ${ }^{27,28}$ radiation. Mass analyzed threshold ionization (MATI) ${ }^{29}$ and pulsed field ionization photoelectron (PFI-PE) ${ }^{30}$ spectra have been reported. Electron momentum spectroscopy has also been employed. ${ }^{31}$ Theoretical predictions for the orbital energies ${ }^{24,25,32}$ and the valence shell photoelectron spectra ${ }^{25,32,33}$ have been obtained.

\section{THEORETICAL APPROACH AND COMPUTATIONAL DETAILS}

\section{A. Framework for treating nuclear dynamics}

In the present work we study the vibrational structure associated with the $\left(\tilde{\mathrm{A}}^{2} \mathrm{~B}_{2}\right.$ $\left.\tilde{\mathrm{B}}^{2} \mathrm{~A}_{1}-\tilde{\mathrm{C}}^{2} \mathrm{~A}_{2}\right)$ and the $\left(\tilde{\mathrm{D}}^{2} \mathrm{~B}_{1}-\tilde{\mathrm{E}}^{2} \mathrm{~B}_{2}\right)$ state photoelectron band systems within the framework of a general multistate multimode vibronic coupling problem, as described by Köppel et al. ${ }^{6}$ Moreover, the actual computational protocol for the two-state problem, ( $\left.\tilde{\mathrm{D}}^{2} \mathrm{~B}_{1}-\tilde{\mathrm{E}}^{2} \mathrm{~B}_{2}\right)$, closely follows that described by Trofimov et al. ${ }^{8}$ For the three-state problem $\left(\tilde{\mathrm{A}}^{2} \mathrm{~B}_{2}-\tilde{\mathrm{B}}^{2} \mathrm{~A}_{1}-\tilde{\mathrm{C}}^{2} \mathrm{~A}_{2}\right)$, the extension of this protocol is straightforward. For this reason we only outline the approach being used, while more details can be found in Ref. 8 .

In the present LVC approach ${ }^{6,14}$ the final vibronic states $\left|\Psi_{m}\right\rangle$ are expanded in a basis of diabatic electronic states $\left|\Phi_{i}\right\rangle:^{34}$

$$
\left|\Psi_{m}(\mathbf{r}, \mathbf{Q})\right\rangle=\sum_{i}\left|\chi_{i m}(\mathbf{Q})\right\rangle\left|\Phi_{i}(\mathbf{r}, \mathbf{Q})\right\rangle
$$

where the summation runs over the set of $N$ vibronically coupled cationic electronic states, and $\mathbf{r}$ and $\mathbf{Q}$ denote the electronic and nuclear coordinates, respectively. The vibrational wavefunctions $\left|\chi_{i m}\right\rangle$ and the corresponding energy levels $\varepsilon_{m}$ are then determined from the eigenvalue equation

$$
\hat{\mathbf{H}} \chi_{m}=\varepsilon_{m} \chi_{m}
$$


where $\hat{\mathbf{H}}$ is the $N$-dimensional model Hamiltonian with elements:

$$
(\hat{\mathbf{H}})_{i j}=\left\{\begin{array}{c}
\hat{H}_{0}+E_{i}+\sqrt{2} \sum_{s \in g} \kappa_{i}^{s} Q_{s}, \quad i=j \\
\sqrt{2} \sum_{s \in u} \lambda_{i j}^{s} Q_{s}, \quad i \neq j
\end{array}\right.
$$

Here $E_{i}$ denote vertical ionization energies associated with the states $\left|\Phi_{i}\right\rangle, \kappa_{i}^{s}$ and $\lambda_{i j}^{s}$ are the intrastate and interstate coupling constants, respectively, and $Q_{s}$ are the dimensionless normal coordinates associated with the totally symmetric $(g)$ or non-totally symmetric $(u)$ normal modes $v_{s} .{ }^{35}$ The Hamiltonian

$$
\hat{H}_{0}=\frac{1}{2} \sum_{s \in g, u} \omega_{s}\left(-\frac{\partial^{2}}{\partial Q_{s}^{2}}+Q_{s}^{2}-1\right)
$$

refers to the electronic ground state $\left|\Phi_{0}\right\rangle$ and describes $M$ non-interacting harmonic oscillators with frequencies $\omega_{s}$. The Hamiltonian (3) introduces the coupling between the electronic states via the potential energy, which is subject to a Taylor expansion through linear terms in the nuclear coordinates.

The Eq. (2) is solved variationally. To this end the vibrational wave functions $\left|\chi_{\text {im }}\right\rangle$ are expanded in terms of the $\hat{H}_{0}$ eigenstates $\left|n_{1} \ldots n_{M}\right\rangle$ :

$$
\left|\chi_{i m}\right\rangle=\sum_{n_{1} \cdots n_{M}} C_{i m}^{n_{1} \cdots n_{M}}\left|n_{1} \ldots n_{M}\right\rangle
$$

where the summation is performed over all possible combinations of harmonic oscillator quantum numbers $n_{s}$ associated with individual modes.

The spectral function describing the transition from the zero vibrational level $\left|\chi_{00}\right\rangle$ of the neutral electronic ground state $\left|\Phi_{0}\right\rangle$ into the manifold of vibronically interacting cationic states $\left|\Phi_{i}\right\rangle$ is given, to a good approximation, ${ }^{8}$ by the expression:

$$
P(E)=\sum_{m} \sum_{i}\left|\left\langle\chi_{00} \mid \chi_{i m}\right\rangle\right|^{2} \delta\left(E-\varepsilon_{m}\right)
$$

If the cationic states are non-interacting (that is, when all $\lambda_{i j}^{s}=0$ ) the spectral function is given by the following analytical expression: ${ }^{6}$ 


$$
P(E)=\sum_{i} \sum_{n_{1} \ldots n_{g}}\left|\left\langle 0 \ldots 0 \mid n_{1} \ldots n_{g}\right\rangle\right|^{2} \delta\left(E-E_{i}+\sum_{s \in g} \omega_{s}\left(a_{i s}-n_{s}\right)\right)
$$

In Eq. (7) each progression of the $i$-th electronic state represents a spectrum characterized by a Poisson intensity distribution (Poisson spectrum), the Franck-Condon factors being given by

$$
\left|\left\langle 0 \ldots 0 \mid n_{1} \ldots n_{g}\right\rangle\right|^{2}=\prod_{s \in g} \frac{\left(a_{i s}\right)^{n_{s}}}{n_{s} !} e^{-a_{i s}}
$$

where

$$
a_{i s}=\left(\kappa_{i}^{s} / \omega_{s}\right)^{2}
$$

is the so-called Poisson (or vibrational strength) parameter.

In order to accomplish the LVC calculations, the parameters $E_{i}, \omega_{s}, \kappa_{i}^{s}, \lambda_{i j}^{s}$ have to be identified. While $E_{i}$ and $\omega_{s}$ can readily be taken from $a b$ initio calculations, special procedures have to be used to determine the coupling constants $\kappa_{i}^{s}$ and $\lambda_{i j}^{s}$. The main idea here is to fit the potentials provided by the potential energy part of the model Hamiltonian (Eq. (3)) to the potential energy surfaces obtained from $a b$ initio calculations. In most cases the explicit fitting is not required, and the coupling constants can be calculated using the following expressions: ${ }^{6,8}$

$$
\begin{aligned}
& \kappa_{i}^{s}=\frac{1}{\sqrt{2}}\left(\frac{\partial E_{i}}{\partial Q_{s}}\right)_{\mathbf{Q}_{0}}, \quad s \in g \\
& \lambda_{i j}^{s}=\frac{1}{\sqrt{2}}\left(\frac{1}{2 \Delta_{s}}\right)\left(\left[E_{i}\left(\mathbf{Q}_{s}\right)-E_{j}\left(\mathbf{Q}_{s}\right)\right]^{2}-\left[E_{i}\left(\mathbf{Q}_{0}\right)-E_{j}\left(\mathbf{Q}_{0}\right)\right]^{2}\right)^{1 / 2}, \quad s \in u
\end{aligned}
$$

where $E_{i}(\mathbf{Q})$ denotes the ionization energy of the $i$-th cationic state at the nuclear configuration $\mathbf{Q}$, and the nuclear configurations $\mathbf{Q}_{s}=\mathbf{Q}_{0} \pm \Delta_{s}$ are obtained by taking the step $\pm \Delta_{S}$ from the equilibrium ground state configuration $\mathbf{Q}_{0}$ along the dimensionless normal coordinate $Q_{s} \cdot{ }^{35}$ The normal modes required for the evaluation of the coupling constants were derived from the Cartesian normal modes computed, together with $\omega_{s}$, using the MP2 method. ${ }^{35}$ The ionization energies $E_{i}(\mathbf{Q})$ at various nuclear configurations were obtained using the OVGF method. ${ }^{15-17}$ The step $\Delta_{\mathrm{s}}=0.5$ was used in the calculations. 
For the solution of Eq. (2), appropriate harmonic oscillator basis sets were constructed for each vibronic problem under consideration (see Secs. IVC2 and IVC3), and then the overlaps $\left\langle\chi_{00} \mid \chi_{i m}\right\rangle$ and the eigenvalues $\varepsilon_{m}$ of $\hat{\mathbf{H}}$ were computed using the Lanczos algorithm. ${ }^{36}$ The Lanczos method allows a sufficiently converged spectral envelope to be obtained prior to the full convergence of the individual transitions. This makes the Lanczos method especially useful in spectroscopic applications. In our case, 10000 Lanczos iterations were performed to generate the spectra of each vibronic symmetry. The general multistate vibronic coupling code was used in these computations. ${ }^{37}$ The theoretical spectral envelopes were obtained by convoluting the generated spectra with Lorentzians of $0.011 \mathrm{eV}$ (FWHM). Such a convolution yields line profiles closely matching the characteristics of the peaks observed in the experimental photoelectron spectra.

\section{B. Ground state parameters}

The equilibrium ground state geometrical parameters of neutral dichloroethene, computed in the present work using the MP2 method and the cc-pVTZ basis set, ${ }^{38,39}$ are shown in Table I, together with the experimental data. ${ }^{40}$ While the calculated C-Cl bond length and the $\mathrm{C}=\mathrm{C}-\mathrm{Cl}$ angle are in excellent agreement with the experimental values, the $\mathrm{C}=\mathrm{C}$ bond length is slightly overestimated and the $\mathrm{C}-\mathrm{H}$ bond length somewhat underestimated by the present calculations. There is also a discrepancy between the calculated and the experimental $\mathrm{C}=\mathrm{C}-\mathrm{H}$ angle.

The corresponding ground state vibrational frequencies, calculated in the harmonic approximation using the same level of theory (MP2/cc-pVTZ), are listed in Table II. The calculated frequencies agree fairly well with measurements. ${ }^{42,43}$ As can be seen, the calculations generally overestimate the frequencies by a factor 1.03-1.06. All the ground state calculations were performed with the GAUSSIAN program package. ${ }^{44}$ 


\section{Calculations of the vertical ionization spectra}

The energies $(E)$ and (relative) spectral intensities (pole strengths, $P$ ) of the vertical ionization transitions below $\sim 18 \mathrm{eV}$ were computed using the OVGF method ${ }^{15-17}$ and the ccpVTZ basis set, ${ }^{38,39}$ as implemented in the GAUSSIAN program package. ${ }^{44}$ The same OVGF/cc-pVTZ approach, as discussed in Sec. IIA, was employed for evaluation of the vibronic coupling constants. The OVGF method provides a consistent third-order description of the ionization processes in situations where the orbital picture of ionization ${ }^{45}$ applies. Such processes normally include all the low-lying ionization transitions. For these transitions, an error of less than $0.2-0.3 \mathrm{eV}$, with respect to the experimental ionization energies, can be expected in OVGF calculations employing the cc-pVTZ, or better quality, basis set. Due to its high numerical efficiency, the OVGF method is very well suited to a large series of computations, such as that of the ionization energies at various nuclear configurations carried out in the context of the present work for the determination of the coupling constants (Eqs. (10) and (11)).

In order to calculate the vertical ionization spectrum of dichloroethene for the entire valence shell, including the inner valence region, the third-order algebraic diagrammatic construction $(\mathrm{ADC}(3))$ approximation scheme for the one-particle Green's function ${ }^{15,46,47}$ was employed. The ADC(3) calculations were performed using the cc-pVTZ basis set with Cartesian representation of the d-functions. The ADC(3) approximation not only describes the main "one-hole" $(1 h)$ electronic states through third-order in the residual electronic interaction, as does the OVGF method, but it also accounts for satellite "two-hole oneparticle" $(2 h-1 p)$ electronic states, which are treated through first-order. The ADC(3) method is therefore applicable in situations where the breakdown of the orbital picture of ionization ${ }^{45}$ takes place. The latter phenomenon manifests itself by a strong redistribution of spectral intensity from the main lines to satellites, and often occurs for inner valence transitions. The ADC(3) and OVGF methods have previously proven to be very successful in similar studies of halogenated molecules. ${ }^{48-54}$ 
In addition, the outer valence vertical ionization energies were computed using the EOM-IP-CCSD method, ${ }^{55-58}$ as implemented in the Q-Chem program package. ${ }^{59}$ The ccpVTZ basis set was also used in these calculations.

In all our electronic structure calculations of the ionization spectra, the carbon and chlorine K-shell orbitals and the chlorine L-shell orbitals were kept frozen. The vertical ionization spectra were computed using the equilibrium ground state structural parameters of dichloroethene obtained from the full geometry optimization at the MP2/cc-pVTZ level of theory (see Sec. IIB). The ADC(3) calculations were performed using the original code ${ }^{60}$ linked to the GAMESS ab initio program package. ${ }^{61,62}$ Theoretical photoelectron spectra were constructed from the $\mathrm{ADC}(3)$ results by convoluting the calculated data with Lorentzians of $0.4 \mathrm{eV}(\mathrm{FWHM})$. The Molden software ${ }^{63}$ was used to plot the Hartree-Fock (HF) molecular orbitals (MOs).

\section{EXPERIMENTAL APPARATUS AND PROCEDURE}

The photoelectron spectra were recorded with a VG Scienta R4000 hemispherical electron energy analyzer mounted on the soft X-ray undulator-based PLÉIADES beamline at the SOLEIL synchrotron radiation facility. Detailed descriptions of the beamline and station instrumentation have been reported previously, ${ }^{64,65}$ so only a summary is given here.

Synchrotron radiation, emitted by an electromagnetic undulator, is dispersed by a modified Petersen type monochromator, ${ }^{66}$ incorporating varied line spacing and varied groove depth gratings, and delivered into the electron spectrometer. The spectrometer is mounted in a fixed position such that the electron detection axis lies perpendicular to the storage ring orbital plane. The undulator allows the plane of the linearly polarized radiation to be chosen to lie either parallel or perpendicular to the orbital plane. The electron spectra were recorded using an analyzer pass energy of $10 \mathrm{eV}$. The photoelectron anisotropy parameters, $\beta$, characterizing the angular distribution, were obtained from spectra recorded using parallel and perpendicularly polarized radiation, as described previously. ${ }^{65}$ The electron spectra were corrected for the transmission efficiency of the analyzer as a function of kinetic energy. ${ }^{67}$ 
The electron binding energy scale was calibrated by comparing a simulation, which included hot-band excitations, of the $\tilde{\mathrm{X}}^{2} \mathrm{~B}_{1}$ state photoelectron band ${ }^{68}$ with the adiabatic ionization energy determined in the MATI experiment.

\section{RESULTS AND DISCUSSION}

\section{A. Overview of the electronic configuration and molecular orbital character}

Our HF/cc-pVTZ calculations, performed in the $\mathrm{C}_{2 \mathrm{v}}$ molecular point group, predict the following ground state valence shell electronic configuration for dichloroethene:

Inner valence : $6 a_{1}^{2} 6 b_{2}^{2} 7 a_{1}^{2} 7 b_{2}^{2}$

Outer valence : $8 a_{1}^{2} 9 a_{1}^{2} 8 b_{2}^{2} 2 b_{1}^{2} 2 a_{2}^{2} 10 a_{1}^{2} 9 b_{2}^{2} 3 b_{1}^{2}$

The $1 b_{2}^{2} 1 a_{1}^{2} 2 a_{1}^{2} 2 b_{2}^{2} 3 b_{2}^{2} 3 a_{1}^{2} 4 b_{2}^{2} 4 a_{1}^{2} 1 a_{2}^{2} 1 b_{1}^{2} 5 b_{2}^{2} 5 a_{1}^{2}$ orbitals belong to the core and represent the K-shell orbitals of carbon and chlorine and the L-shell orbitals of chlorine. The character of the outer valence orbitals can be assessed from the Mulliken atomic populations ${ }^{69}$ given in Table III. The eight highest occupied orbitals are plotted in Figure 2 using the results from our HF/cc-pVTZ calculations.

The highest occupied orbital, $3 b_{1}$, can be assigned as a $\pi$-type $\mathrm{C}=\mathrm{C}$ double bond. However, the Mulliken populations show that the chlorine character in the $3 b_{1}$ orbital is substantial and almost comparable with the carbon content. This implies that the $3 b_{1}$ orbital only nominally represents the carbon-carbon double bond and has features similar to that of the $\pi$-type orbital describing the chlorine lone-pairs (Figure 2).

A related orbital, $2 b_{1}$, is constructed in a manner similar to the $3 b_{1}$ orbital (Figure 2). According to the Mulliken populations, this orbital should nominally be referred to as a chlorine lone-pair $\left(\pi_{\mathrm{Cl}} \mathrm{LP}\right)$, since the chlorine character slightly exceeds the carbon character. The difference between the $3 b_{1}$ and $2 b_{1}$ MOs can be understood from the plots shown in Figure 2. For the $3 b_{1}$ orbital a nodal plane occurs between the $\mathrm{C}$ and $\mathrm{Cl}$ atoms, while the $2 \mathrm{~b}_{1}$ orbital is fully bonding. 
In contrast to the $2 b_{1} \mathrm{MO}$, the $2 \mathrm{a}_{2}$ orbital contains no contribution from the carbons and can be considered as a pure $\pi$-type combination of chlorine lone pairs $\left(\pi_{\mathrm{Cl} L \mathrm{LP}}\right)$.

The $9 \mathrm{~b}_{2}$ and $10 \mathrm{a}_{1} \sigma$-type orbitals are also practically pure chlorine lone-pair orbitals $\left(\sigma_{\mathrm{Cl}} \mathrm{LP}\right)$. As can be seen from the plots in Figure 2, these orbitals differ only in the sign of the combination of the atomic chlorine p-orbitals.

According to the present analysis (Table III and Figure 2), the $8 \mathrm{~b}_{2}$ and $9 \mathrm{a}_{1}$ orbitals are also chlorine $\sigma$-type lone-pairs and are involved in the $\mathrm{C}$ - $\mathrm{Cl}$ bonding. The 8 $\mathrm{a}_{1}, \sigma$-type orbital, describes bonding between all the atoms.

\section{B. Assignment of the photoelectron spectrum of dichloroethene}

The present HF, OVGF, ADC(3) and EOM-IP-CCSD results for the vertical outer valence ionization transitions, obtained with the cc-pVTZ basis sets, are listed in Table IV together with the experimental values. The OVGF, ADC(3) and EOM-IP-CCSD vertical ionization energies for all transitions are remarkably consistent with each other, and in good agreement with the experimental values.

The orbital picture of ionization is fulfilled for the six lowest ionization transitions. According to our $\mathrm{ADC}(3)$ calculations, the lowest $2 h$-1p-satellite appears at $14.58 \mathrm{eV}$ $(P \sim 0.01)$ and represents a transition to a $\pi-\pi^{*}$ excited state of the cation having ${ }^{2} \mathrm{~A}_{2}$ symmetry. This excited state is predicted to have a dominant electronic configuration of $3 b_{1}{ }^{-2} 3 a_{2}$ and to acquire its intensity from the $2 a_{2}\left(\pi_{C L} L P\right)$ orbital. The next satellite occurs at $15.68 \mathrm{eV}(P \sim 0.27)$ and is a shake-down transition with the final state ${ }^{2} \mathrm{~A}_{1}\left(9 \mathrm{~b}_{2}{ }^{-1} 2 \mathrm{~b}_{1}{ }^{-1} 3 \mathrm{a}_{2}\right)$. Our $\operatorname{ADC}(3)$ calculations predict that this satellite is related to the ionization of the $9 \mathrm{a}_{1}(\sigma) \mathrm{MO}$ whose main $(1 h-)$ line appears at $15.79 \mathrm{eV}(P \sim 0.60)$. Satellites become more numerous at higher energy, but the orbital picture of ionization ${ }^{45}$ generally holds below $\sim 20 \mathrm{eV}$ (Figure 3), thereby allowing the experimental spectrum to be interpreted in terms of the main lines. Hence, the assignment of the observed structure is fairly straightforward. 
The theoretical spectrum obtained using the results of our ADC(3)/cc-pVTZ calculations is shown in Figure 3, together with the valence shell photoelectron spectrum recorded at a photon energy of $80 \mathrm{eV}$, for electrons ejected parallel to the plane of polarization of the linearly polarized radiation. The first band, due to the $\left(3 \mathrm{~b}_{1}\right)^{-1} \tilde{\mathrm{X}}^{2} \mathrm{~B}_{1}$ state, displays extensive vibrational structure. The adiabatic transition occurs at $9.659 \mathrm{eV}$, and the center of gravity of the band is located at $\sim 9.8 \mathrm{eV}$. The latter agrees very well with the present ADC(3) vertical ionization energy of $9.75 \mathrm{eV}$.

The group of three closely spaced bands observed in the binding energy range $\sim 11.2-$ $13.1 \mathrm{eV}$, with peaks at $11.70,12.06$ and $12.54 \mathrm{eV}$, can be assigned to the $\left(9 \mathrm{~b}_{2}\right)^{-1} \tilde{\mathrm{A}}^{2} \mathrm{~B}_{2},\left(10 \mathrm{a}_{1}\right)^{-}$ ${ }^{1} \tilde{\mathrm{B}}^{2} \mathrm{~A}_{1}$ and $\left(2 \mathrm{a}_{2}\right)^{-1} \tilde{\mathrm{C}}^{2} \mathrm{~A}_{2}$ states. The vertical ionization energies for these states, obtained in our ADC(3) calculations, are 11.64, 11.98 and $12.48 \mathrm{eV}$, respectively.

According to our ADC(3) results, the complicated structure observed between $\sim 13.4$ and $14.6 \mathrm{eV}$ originates from the closely spaced $\left(2 \mathrm{~b}_{1}\right)^{-1} \tilde{\mathrm{D}}^{2} \mathrm{~B}_{1}$ and $\left(8 \mathrm{~b}_{2}\right)^{-1} \tilde{\mathrm{E}}^{2} \mathrm{~B}_{2}$ states, with calculated vertical ionization energies of 13.93 and $14.21 \mathrm{eV}$, respectively. These energies are in reasonable accord with those of $\sim 13.80$ and $14.21 \mathrm{eV}$ for the peak maximum and shoulder observed in the experimental spectrum. The predicted $3 b_{1}{ }^{-2} 3 a_{2}$ satellite, calculated to occur at $14.58 \mathrm{eV}$, may contribute to the width of this band.

The band occurring at $\sim 15.70 \mathrm{eV}$ in the experimental spectrum is due to ionization of the $9 \mathrm{a}_{1}$ orbital. The calculations predict a vertical ionization energy of $15.79 \mathrm{eV}$, and also suggest that this band is influenced by satellites on the low binding energy side of the main line.

Our theoretical results (Figure 3), indicate that satellites also contribute to the next two bands in the experimental spectrum, due to the $8 \mathrm{a}_{1}(\sigma)^{-1}$ and $7 \mathrm{~b}_{2}(\sigma)^{-1}$ states, with maxima at 16.9 and $18.9 \mathrm{eV}$, respectively. The corresponding peak maxima at 17.38 and $19.10 \mathrm{eV}$, respectively, are shifted slightly from the experimental values, apparently due to the increased $2 h-1 p$ - character of the final states. 
The bands at binding energies above $20 \mathrm{eV}$ are affected substantially by break-down phenomena and can no longer be described by the orbital picture of ionization. ${ }^{45}$ Here, the experimental spectrum becomes increasingly diffuse since groups of overlapping satellite states, covering a wide energy range, replace the dominant main line transitions. Although the structure in this region is extremely complex, a few bands can be assigned. The prominent maximum observed at $22.56 \mathrm{eV}$ can be attributed to states gaining their intensity from the ionization of the $7 \mathrm{a}_{1}$ orbital. A further calculated peak maximum at $23.38 \mathrm{eV}$ (Figure 3) corresponds to an intense satellite $(P=0.34)$ of a complex nature containing contributions from configurations such as $7 \mathrm{~b}_{2}^{-1} 3 \mathrm{~b}_{1}^{-1} 3 \mathrm{a}_{2}$.

At higher energy, the spectrum shows two broad peaks centered at $\sim 25.4$ and $26.9 \mathrm{eV}$. The interpretation of these peaks is less conclusive as the theoretical spectral profile appears more structured than the experimental spectrum. However, it seems that the two peaks are mainly due to transitions related to the $6 b_{2}$ and $6 a_{1}$ orbitals.

\section{Vibrational structure of the lowest photoelectron bands}

As has been shown in Sec. IVB, our electronic structure calculations provide a reliable description of the cationic states responsible for the photoelectron bands observed below the onset, at $\sim 14.5 \mathrm{eV}$, of the $2 h-1 p$-satellites. According to our results, six cationic states occur in the binding energy range below this onset. The lowest of these states gives rise to an isolated band between $\sim 9.6$ and $10.7 \mathrm{eV}$, assigned to the $\tilde{\mathrm{X}}^{2} \mathrm{~B}_{1}$ state. The next three states, $\tilde{\mathrm{A}}^{2} \mathrm{~B}_{2}$, $\tilde{\mathrm{B}}^{2} \mathrm{~A}_{1}$ and $\tilde{\mathrm{C}}^{2} \mathrm{~A}_{2}$, result in a complicated band system lying in the binding energy range $\sim 11.2$ to $13.1 \mathrm{eV}$. Another complicated band system, observed between $\sim 13.4$ and $14.6 \mathrm{eV}$, is due to the $\tilde{\mathrm{D}}^{2} \mathrm{~B}_{1}$ and $\tilde{\mathrm{E}}^{2} \mathrm{~B}_{2}$ states. Only the $\tilde{\mathrm{X}}^{2} \mathrm{~B}_{1}$ state band exhibits extended vibrational progressions. The remaining features, belonging to the $\left(\tilde{\mathrm{A}}^{2} \mathrm{~B}_{2}-\tilde{\mathrm{B}}^{2} \mathrm{~A}_{1}-\tilde{\mathrm{C}}^{2} \mathrm{~A}_{2}\right)$ and the $\left(\tilde{\mathrm{D}}^{2} \mathrm{~B}_{1}\right.$ $\tilde{\mathrm{E}}^{2} \mathrm{~B}_{2}$ ) band systems, are rather diffuse and display irregular vibrational structure. This suggests that the adiabatic approximation may not hold for these two band systems, thereby implying that a theoretical approach taking into account vibronic coupling and non-adiabatic 
effects has to be employed to obtain a proper explanation of the observed vibrational structure.

\section{The $\tilde{X}^{2} B_{1}$ state}

At the equilibrium ground state geometry of neutral dichloroethene, the lowest cationic state is separated from the higher lying states by a substantial energy interval $(\sim 1.9$ $\mathrm{eV}$, Table IV). Therefore the adiabatic approximation should be valid for the $\tilde{\mathrm{X}}^{2} \mathrm{~B}_{1}$ state, with the nuclear dynamics being described in terms of a single potential energy surface.

The key characteristics, such as the equilibrium geometry and the vibrational frequencies, of the $\tilde{\mathrm{X}}^{2} \mathrm{~B}_{1}$ state, as calculated in the present work at the MP2/cc-pVTZ level of theory, are listed in Tables I and II, respectively. The corresponding data for the neutral ground state are also given. As can be seen, the most important changes in the geometry of the $\tilde{\mathrm{X}}^{2} \mathrm{~B}_{1}$ state in comparison with that of the ground state include a slight increase in the $\mathrm{C}=\mathrm{C}$ bond length and a decrease of about the same amount in the $\mathrm{C}-\mathrm{Cl}$ bond length (Table I). Thus, except for the $v_{2}\left(a_{1}\right), v_{4}\left(a_{1}\right)$ and $v_{7}\left(a_{2}\right)$ modes, with the first two being associated with changes in the $\mathrm{C}=\mathrm{C}$ and $\mathrm{C}-\mathrm{Cl}$ bond lengths, there are only minor changes in the frequencies of most of the harmonic vibrations. The frequencies for the $v_{2}$ and $v_{7}$ modes are strongly reduced in the $\tilde{\mathrm{X}}^{2} \mathrm{~B}_{1}$ state (Table II). A pronounced modification to the $\mathrm{C}=\mathrm{C}$ bond length in the $\tilde{\mathrm{X}}^{2} \mathrm{~B}_{1}$ state is also predicted by the large intrastate coupling constant $\kappa$ obtained for the $v_{2}$ mode (Table V). The elongation of the $\mathrm{C}=\mathrm{C}$ bond can be expected since the $\tilde{\mathrm{X}}^{2} \mathrm{~B}_{1}$ state is obtained by ionization of the $3 b_{1}(\pi)$ orbital which is bonding with respect to the carbon atoms. The antibonding character of this orbital with respect to the carbon-chlorine pair of atoms is responsible for the reduction in the $\mathrm{C}-\mathrm{Cl}$ bond lengths (Sec. IVA, Figure 2), which leads to a noticeable increase in the frequency of the $v_{4}$ mode.

The intrastate coupling constants $\kappa$ for the totally symmetric modes in the $\tilde{\mathrm{X}}^{2} \mathrm{~B}_{1}$ state (Table V), and the corresponding ground state vibrational frequencies (Table II) were used as parameters in the LVC model to compute the Poisson vibrational spectrum, according to 
Eq. (7). The calculated Poisson spectrum (Figure 4(c)) qualitatively agrees with the experimental spectrum (Figure 4(a)), but some discrepancies in the frequencies and intensities of the vibrational excitations are apparent. Also, the LVC/Poisson spectrum is shifted in energy with respect to the experimental profile. The calculated adiabatic transition energy $(9.43 \mathrm{eV})$ for the $\tilde{\mathrm{X}}^{2} \mathrm{~B}_{1}$ state is noticeably lower than the experimental value of $9.659 \mathrm{eV}$ (Table VI).

Since the adiabatic approximation holds for the $\tilde{\mathrm{X}}^{2} \mathrm{~B}_{1}$ state, a more rigorous approach can be employed to generate the theoretical spectrum. The Franck-Condon factors can be computed at a level beyond the LVC approximation using the harmonic potential energy surfaces obtained at the MP2/cc-pVTZ level of theory for the neutral ground state and the $\tilde{\mathrm{X}}^{2} \mathrm{~B}_{1}$ cationic state (Fig 4(b)). The explicit evaluation of the Franck-Condon factors was performed using the pre-screening scheme $e^{70,71}$ as implemented in the GAUSSIAN package. ${ }^{44}$ In contrast to the LVC/Poisson spectrum, the final state $\left(\tilde{\mathrm{X}}^{2} \mathrm{~B}_{1}\right)$ vibrational frequencies were used to generate the spectral intensities. The Franck-Condon spectrum obtained in this way (Figure 4(b)) is in excellent agreement with the experimental data (Figure 4(a)), and the predicted adiabatic transition energy of $9.64 \mathrm{eV}$ is in accord with the measured value. This closer agreement reflects the importance of the more accurate vibrational frequencies and of the improved description of the vibrational modes in the modeling of the spectrum. We note also that the Duschinsky effect ${ }^{72}$ of the normal mode mixing in the final state, which is not treated in the LVC model, is now taken into account.

The present theoretical results indicate that the $\tilde{\mathrm{X}}^{2} \mathrm{~B}_{1}$ state photoelectron spectrum can be explained satisfactorily in terms of only the totally symmetric modes, in agreement with the Franck-Condon principle. According to our predictions, the most active modes are $v_{2}$ through to $v_{5}($ Table $\mathrm{V}$ ), and the observed peaks can be assigned to excitations involving these modes (Figure 2). The influence of hot-bands on the vibrational structure has been considered in detail by Powis et al. ${ }^{68}$ 


\section{The $\tilde{A}^{2} B_{2}, \tilde{B}^{2} A_{1}$ and $\tilde{C}{ }^{2} A_{2}$ states}

Since the vertical energy gap between the $\tilde{\mathrm{A}}^{2} \mathrm{~B}_{2}, \tilde{\mathrm{B}}^{2} \mathrm{~A}_{1}$ and $\tilde{\mathrm{C}}^{2} \mathrm{~A}_{2}$ states is rather small (Table IV), vibronic coupling between these states seems plausible. In this work we treat all three states within the LVC model, where we assume that they are coupled via the non-totally symmetric $b_{1}, a_{2}$, and $b_{2}$ modes $\left(\mathrm{B}_{2} \times \mathrm{A}_{2} \times b_{1} \supset \mathrm{A}_{1}, \mathrm{~A}_{2} \times \mathrm{A}_{1} \times a_{2} \supset \mathrm{A}_{1}, \mathrm{~B}_{2} \times \mathrm{A}_{1} \times b_{2} \supset \mathrm{A}_{1}\right)$. The LVC model parameters were evaluated as described in Sec. II, and the resulting intrastate coupling constants $\kappa$ and the interstate coupling constants $\lambda$ are given in Tables $V$ and VII, respectively.

The intrastate coupling constants show that the low frequency $v_{5}\left(a_{1}\right)$ mode plays an extremely important role in the nuclear dynamics of the $\tilde{\mathrm{A}}^{2} \mathrm{~B}_{2}, \tilde{\mathrm{B}}^{2} \mathrm{~A}_{1}$ and $\tilde{\mathrm{C}}^{2} \mathrm{~A}_{2}$ states. This mode describes the totally symmetric deformation vibration of the carbon and chlorine atoms, often referred to as a scissoring of the chlorine atoms. The very large Poisson parameters (Eq. (9)) of 10.9, 5.1 and 2.4 obtained for the $\tilde{\mathrm{A}}^{2} \mathrm{~B}_{2}, \tilde{\mathrm{B}}^{2} \mathrm{~A}_{1}$ and $\tilde{\mathrm{C}}^{2} \mathrm{~A}_{2}$ states, respectively, along this mode, indicate that the $v_{5}$ mode is highly excited upon ionization to these states and actively participates in the tuning of the conical intersections between the corresponding potential energy surfaces. The next, although much less important, $a_{1}$ mode influencing the dynamics is the $\mathrm{C}-\mathrm{Cl}$ stretching vibration $v_{4}$. In contrast to the $\tilde{\mathrm{X}}^{2} \mathrm{~B}_{1}$ state dynamics, the $\mathrm{C}=\mathrm{C}$ stretching mode $v_{2}$ plays only a minor role in the $\tilde{\mathrm{A}}^{2} \mathrm{~B}_{2}, \tilde{\mathrm{B}}^{2} \mathrm{~A}_{1}$ and $\tilde{\mathrm{C}}^{2} \mathrm{~A}_{2}$ state dynamics. This is not unexpected since these three states are obtained by ionization of non-bonding chlorine lone-pair orbitals (Table III). According to the $\kappa$ constants and the Poisson parameters, the totally symmetric $v_{1}$ and $v_{3}$ hydrogen vibrations are the least excited modes for the manifold of cationic states under consideration and can be discarded in the vibronic modeling. The present vibronic model used to calculate the spectrum therefore included the $v_{5}, v_{4}$, and $v_{2} a_{1}$ modes. The harmonic oscillator basis set $\left|n_{1} \ldots n_{M}\right\rangle$ adopted for the variational calculations (Eq. (5)) comprised the corresponding functions with quantum numbers $n_{5}, n_{4}$, and $n_{2}$ up to 50,8 and 2 , respectively.

When the intrastate coupling constants $\kappa$, the ground state vibrational frequencies $\omega_{s}$ and the vertical ionization energies $E_{i}$ are known, the LVC formalism can be used to evaluate 
the adiabatic transition energies and the minimal energies of the conical intersections between the potential energy surfaces of the interacting states. ${ }^{6}$ The results obtained in this work for the $\tilde{\mathrm{A}}^{2} \mathrm{~B}_{2}, \tilde{\mathrm{B}}^{2} \mathrm{~A}_{1}$ and $\tilde{\mathrm{C}}^{2} \mathrm{~A}_{2}$ states are listed in Table VI. According to the present adiabatic transition energies $E_{0-0}$ the adiabatic minimum of the $\tilde{\mathrm{A}}^{2} \mathrm{~B}_{2}$ state is well below the minimum of the $\tilde{\mathrm{B}}^{2} \mathrm{~A}_{1}$ state, and also below the lowest energy of the conical intersection with the $\tilde{\mathrm{B}}^{2} \mathrm{~A}_{1}$ state. This means that the adiabatic approximation should hold for at least the lowest vibrational levels of the $\tilde{\mathrm{A}}{ }^{2} \mathrm{~B}_{2}$ state which are well below the conical intersection. The situation described above can be seen from the potential energy curves for the $\tilde{\mathrm{A}}^{2} \mathrm{~B}_{2}, \tilde{\mathrm{B}}^{2} \mathrm{~A}_{1}$ and $\tilde{\mathrm{C}}^{2} \mathrm{~A}_{2}$ states along the most active totally symmetric normal coordinate $Q_{5}$ (Figure $\mathrm{S} 1$ of the Supplementary Material). The curves illustrate the set of potential minima and these minima may be compared to those of the conical intersection seams.

The situation is more complex for the $\tilde{\mathrm{B}}^{2} \mathrm{~A}_{1}$ state as here the conical intersection occurs in the vicinity of its adiabatic minimum. This means that all vibrational levels associated with the $\tilde{\mathrm{B}}^{2} \mathrm{~A}_{1}$ state potential energy surface are affected, and that the nuclear dynamics proceed on the coupled $\tilde{\mathrm{A}}^{2} \mathrm{~B}_{2}$ and $\tilde{\mathrm{B}}^{2} \mathrm{~A}_{1}$ potential energy surfaces in a nonadiabatic manner. A similar situation occurs in the $\tilde{\mathrm{C}}^{2} \mathrm{~A}_{2}$ state. Thus, the $\tilde{\mathrm{B}}^{2} \mathrm{~A}_{1}$ and $\tilde{\mathrm{C}}^{2} \mathrm{~A}_{2}$ state photoelectron bands should be affected strongly by non-adiabatic effects. ${ }^{6}$ The intersection between the potential energy surfaces associated with the $\tilde{\mathrm{A}}^{2} \mathrm{~B}_{2}$ and $\tilde{\mathrm{C}}^{2} \mathrm{~A}_{2}$ states takes place at much higher energy and therefore should not be relevant to the spectrum (Table VI, Figure S1).

The interstate coupling constants $\lambda$ characterizing the interactions amongst the $\tilde{\mathrm{A}}^{2} \mathrm{~B}_{2}$, $\tilde{\mathrm{B}}^{2} \mathrm{~A}_{1}$ and $\tilde{\mathrm{C}}^{2} \mathrm{~A}_{2}$ states, via various non-totally symmetric modes, are given in Table VII. Here, the derivation of the constants was complicated by the specific shape of the potential energy surface of the $\tilde{\mathrm{B}}^{2} \mathrm{~A}_{1}$ state along the $v_{9}, v_{11}$, and $v_{12}$ modes, all of $b_{2}$ symmetry (Table II). According to our ionization energies computed for the molecular geometries distorted along the corresponding vibrational coordinates, the potential energy surface of the $\tilde{\mathrm{B}}^{2} \mathrm{~A}_{1}$ state is flatter than that of the neutral ground state, whereas a steeper potential energy surface is expected within the LVC approach for non-zero coupling to the potential energy surface of 
the lower state $\left(\tilde{\mathrm{A}}^{2} \mathrm{~B}_{2}\right.$ in our case). Thus, the $\tilde{\mathrm{B}}^{2} \mathrm{~A}_{1}$ state potential energy surface obtained in our ab initio OVGF calculations cannot be fitted to a LVC model and Eq. (11) cannot be used to compute the coupling constants $\lambda$. In such situations, a higher-level model has to be adopted or an additional ${ }^{2} \mathrm{~B}_{2}$ state has to be introduced in order to make the fitting possible. However, since the lower ( $\tilde{\mathrm{A}}^{2} \mathrm{~B}_{2}$ state) potential energy surface has the expected shape (which is also flatter than that of the neutral ground state), reflecting the existence of a nonzero coupling along the $v_{9}\left(b_{2}\right), v_{11}\left(b_{2}\right)$, and $v_{12}\left(b_{2}\right)$ modes, one can try to estimate this coupling in an approximate manner. To do this we stay within the LVC level of approximation and assume that the potential energy surface of the $\tilde{\mathrm{B}}^{2} \mathrm{~A}_{1}$ state has the same characteristics as those of the neutral ground state. This means that the ionization energies $E_{i}(\mathbf{Q})$ of the $\tilde{\mathrm{B}}^{2} \mathrm{~A}_{1}$ state do not change along the $v_{9}, v_{11}$, and $v_{12}$ coordinates, and that Eq. (11) can be applied to compute $\lambda_{i j}^{s}$. The coupling constants $\lambda$ obtained in this way (Table VII) can then be considered as upper bound estimates describing the coupling between the $\tilde{\mathrm{A}}^{2} \mathrm{~B}_{2}$ and $\tilde{\mathrm{B}}^{2} \mathrm{~A}_{1}$ states. These $\lambda$ constants are also used in our LVC model to obtain a preliminary description of the coupling and a qualitative level description of the spectrum. The extent of the approximation of putting the $\tilde{\mathrm{A}}^{2} \mathrm{~B}_{2}$-state potential energy curve equal to that of the neutral ground state can be judged explicitly from the respective plots shown in Figure S2 of the Supplementary Material. A proper treatment of the nuclear dynamics considered here would require the setting up of an extended model followed by an appropriate evaluation of all the parameters entering the model.

According to the ratio $\left|\lambda_{i j}^{s} / \omega_{s}\right|<1$ (Tables VII and II), the vibronic coupling strength in the system comprising the $\tilde{\mathrm{A}}^{2} \mathrm{~B}_{2}, \quad \tilde{\mathrm{B}}^{2} \mathrm{~A}_{1}$ and $\tilde{\mathrm{C}}^{2} \mathrm{~A}_{2}$ states can be characterized approximately as moderate (a strong coupling assumes $\left|\lambda_{i j}^{s} / \omega_{s}\right|>>1$ ). ${ }^{6}$ In agreement with this assessment, no double-minimum potential energy surface was predicted by the present LVC model along any of the non-totally symmetric vibrational coordinates. Consequently, none of the $\tilde{\mathrm{A}}^{2} \mathrm{~B}_{2}, \tilde{\mathrm{B}}^{2} \mathrm{~A}_{1}$ or $\tilde{\mathrm{C}}^{2} \mathrm{~A}_{2}$ final cationic states would lead to a molecular structure with broken symmetry (lower than the $\mathrm{C}_{2 \mathrm{v}}$ point group characterizing the neutral ground state). However, this conclusion might change if a more accurate model (and coupling 
constants) could be adopted or if a direct $a b$ initio geometry optimization for the cationic states could be performed using an adequate electronic structure method. Our results indicate that the most important modes are the $v_{10}\left(b_{2}\right), v_{11}\left(b_{2}\right)$ (coupling the $\tilde{\mathrm{A}}^{2} \mathrm{~B}_{2}$, and $\tilde{\mathrm{B}}^{2} \mathrm{~A}_{1}$ states), $v_{6}\left(a_{2}\right)$, and $v_{7}\left(a_{2}\right)$ (coupling the $\tilde{\mathrm{B}}^{2} \mathrm{~A}_{1}$ and $\tilde{\mathrm{C}}^{2} \mathrm{~A}_{2}$ states), and $v_{8}\left(b_{1}\right)$ (coupling the $\tilde{\mathrm{A}}^{2} \mathrm{~B}_{2}$, and $\tilde{\mathrm{C}}^{2} \mathrm{~A}_{2}$ states). These modes were taken into account in the present LVC model and the corresponding harmonic oscillator functions with quantum numbers $n_{10}, n_{11}, n_{6}, n_{7}, n_{8}$ up to $10,10,8,17,10$, respectively, were used to form the basis set $\left|n_{1} \ldots n_{M}\right\rangle$ for calculating the spectrum.

The results of our vibronic modeling of the $\left(\tilde{\mathrm{A}}^{2} \mathrm{~B}_{2}-\tilde{\mathrm{B}}^{2} \mathrm{~A}_{1}-\tilde{\mathrm{C}}^{2} \mathrm{~A}_{2}\right)$ state band system are shown in Figure 5 where the theoretical spectrum accounting for vibronic coupling amongst all three states (Figure 5(b)) is compared with the Poisson spectrum which is obtained using an approximation where vibronic coupling is not treated (Figure 5(c)). The experimental spectrum is also shown (Figure 5(a)). The most striking spectral feature, namely the splitting of the second band, observed between $\sim 11.9$ and $12.3 \mathrm{eV}$, into two strong components, cannot be reproduced assuming non-interacting cationic states, but is reproduced qualitatively by the vibronic coupling calculations. This confirms that the present vibronic treatment has been performed at a suitable level, and it also demonstrates the extent to which vibronic interaction can modify spectral structure.

By comparing the spectra shown in Figure 5 it can be seen that the onset of irregularity in the experimental $\tilde{\mathrm{A}}^{2} \mathrm{~B}_{2}$ state photoelectron band above $11.7 \mathrm{eV}$ can be attributed to an additional vibrational excitation (indicated as red bars in Figure 5(b)) in the vibronic coupling model. This additional motion corresponds to a $b_{2}$ mode on the $\tilde{\mathrm{A}}{ }^{2} \mathrm{~B}_{2}$ state adiabatic potential energy surface, and would thus be forbidden in the Franck-Condon approximation. The Franck-Condon allowed progression in the $a_{1}$ vibrational mode (indicated as green bars) is overlapped at higher ionization energy by the $\tilde{\mathrm{A}}^{2} \mathrm{~B}_{2} \times b_{2}$ vibronic excitation that generates a second dominant vibrational progression. This second progression passes through a maximum near $11.8 \mathrm{eV}$ in the theoretical spectrum and falls off before the energy of the conical intersection is approached at $\sim 11.87 \mathrm{eV}$. More specifically, the dominant mode is $v_{5}$ in both 
the $\tilde{\mathrm{A}}^{2} \mathrm{~B}_{2}$ and the $\tilde{\mathrm{B}}^{2} \mathrm{~A}_{1}$ states. In the former case its excitations yield a conventional FranckCondon progression (green lines in Figure 5(b)). The second progression mentioned above (red lines in Figure 5(b)) can be interpreted as excitations of the $\tilde{\mathrm{A}}^{2} \mathrm{~B}_{2}-\tilde{\mathrm{B}}^{2} \mathrm{~A}_{1}$ coupling modes, namely the $v_{10}\left(\mathrm{CH}\right.$ bend) and $v_{11}(\mathrm{CCl}$ stretch $)$ modes. This discussion implies that in the energy range below $11.9 \mathrm{eV}$ one does not have nonadiabatic coupling effects, but rather effects of vibronic intensity borrowing. The latter effects are possible below the conical intersection [6], which, according to our results for the $\tilde{\mathrm{A}}^{2} \mathrm{~B}_{2}$ and $\tilde{\mathrm{B}}^{2} \mathrm{~A}_{1}$ states, occurs at $11.87 \mathrm{eV}$ (Table VI).

According to our theoretical predictions, the photoelectron bands associated with the $\tilde{\mathrm{B}}^{2} \mathrm{~A}_{1}$ and $\tilde{\mathrm{C}}^{2} \mathrm{~A}_{2}$ states lie mostly within the domain of non-adiabatic nuclear dynamics, where the dynamics proceed on the potential energy surfaces of both states. This leads to highly complex structure in the computed vibronic spectrum and makes the assignment of the vibronic transitions very difficult. The interpretation of the final vibronic states is also complicated by the presently used formalism of diabatic electronic states, necessitating a transformation of the results to the basis of adiabatic states to make them tractable., ${ }^{6,34}$

Our theoretical results are unable to provide an unambiguous explanation for the splitting of the $\tilde{\mathrm{B}}^{2} \mathrm{~A}_{1}$ state band into two distinct components. In the theoretical spectrum, each of these components is formed by a large number of vibronic excitations of a complex nature. These excitations, shown as red bars in Figure 5(b), belong to the $A_{1}$ vibronic symmetry, implying that their final states can be various combinations of $\tilde{\mathrm{B}}^{2} \mathrm{~A}_{1} \times a_{1}, \tilde{\mathrm{A}}{ }^{2} \mathrm{~B}_{2} \times b_{2}$ and $\tilde{\mathrm{C}^{2}} \mathrm{~A}_{2} \times a_{2}$ vibronic states. The $\tilde{\mathrm{B}}{ }^{2} \mathrm{~A}_{1} \times a_{1}$ states, associated with the $\tilde{\mathrm{B}}^{2} \mathrm{~A}_{1}$ state potential energy surface, can be viewed as the normal Franck-Condon states presented in Figure 5(c). The $\tilde{\mathrm{A}}^{2} \mathrm{~B}_{2} \times b_{2}$ states correspond to excitations of the $b_{2}$ modes associated with the lower $\tilde{\mathrm{A}}^{2} \mathrm{~B}_{2}$ state potential energy surface and form, in the $11.9-12.2 \mathrm{eV}$ energy range, a dense quasi-continuum of vibronic states embedded within the $\tilde{\mathrm{B}}^{2} \mathrm{~A}_{1} \times a_{1}$ states. This gives rise to resonance-like broad spectral structures formed by bundles of $\tilde{\mathrm{A}}{ }^{2} \mathrm{~B}_{2} \times b_{2}$ states around the $\tilde{B}^{2} \mathrm{~A}_{1} \times a_{1}$ states. The width of the structures formed in this way reflects the reduced lifetimes of the vibrational levels associated with the $\tilde{\mathrm{B}}^{2} \mathrm{~A}_{1}$ state potential energy surface. These levels 
decay quickly on a femtosecond timescale into the levels associated with the lower $\tilde{\mathrm{A}}^{2} \mathrm{~B}_{2}$ state potential energy surface.

Finally, we propose a tentative, more specific explanation for the double peak structure occurring in the $\tilde{\mathrm{B}}^{2} \mathrm{~A}_{1}$ state photoelectron band based upon the observation that the Poisson spectrum of this state (Figure 5(c)) is formed by two major progressions due to the $5_{0}^{n}$ and $5_{0}^{n} 4_{0}^{m}$ excitations of the $a_{1}$ modes. These progressions are evident in the spectrum and their intensity maxima occur near 11.94 and $12.03 \mathrm{eV}$, respectively. These two energies are roughly the same as those characterizing the two peaks of the $\tilde{\mathrm{B}}^{2} \mathrm{~A}_{1}$ state in the vibronic band (11.96 and 12.01-12.05 eV, respectively) in Figure 5(b) and experimental spectrum (11.96 and 12.02-12.07 eV, respectively) in Figure 5(a). Taking into account the discussion in the preceding paragraph, this correspondence suggests that the formation and shape of the two components of the $\tilde{\mathrm{B}}^{2} \mathrm{~A}_{1}$ state band in the vibronic spectrum reflect the decay of the $5_{0}^{n}$ and $5_{0}^{n} 4_{0}^{m}$ states into the $b_{2}$ levels of the $\tilde{\mathrm{A}}^{2} \mathrm{~B}_{2}$ state potential energy surface.

A shoulder observed in the $\tilde{\mathrm{B}}^{2} \mathrm{~A}_{1}$ state band at $\sim 12.13 \mathrm{eV}$ (Figure 5(a)) can be explained using similar arguments. Inspection of the theoretical spectra allows one to suggest that this feature is related to the $5_{0}^{n} 2_{0}^{1}$ progression in the Poisson spectrum (Figure 5(c)) showing its intensity maximum near $\sim 12.12 \mathrm{eV}$ (the $5_{0}^{4} 2_{0}^{1}$ transition). The decay of these energy levels by the vibronic coupling mechanism, as discussed above, gives rise to the maximum in the vibronic spectrum at $\sim 12.14 \mathrm{eV}$ (Figure 5(b)), correlating with the shoulder in the experimental spectrum.

The diffuse nature of the $\tilde{\mathrm{C}}^{2} \mathrm{~A}_{2}$ state photoelectron band (Figure 5(a)) is explained satisfactorily by the theoretical results which predict an extremely dense manifold of vibronic transitions (Figure 5(b)). The complex character of the transitions forming the spectral envelope reflects the non-adiabatic nuclear dynamics and the complicated decay processes affecting the vibronic states. The vibronic states contributing to the $\tilde{\mathrm{C}}^{2} \mathrm{~A}_{2}$ state photoelectron band are combinations of the totally symmetric vibrational excitations in this state $\left(\tilde{\mathrm{C}}^{2} \mathrm{~A}_{2} \times a_{1}\right)$ and various non-totally symmetric vibrational excitations associated with the lower electronic states $\left(\tilde{\mathrm{B}}^{2} \mathrm{~A}_{1} \times a_{2}\right.$ and $\left.\tilde{\mathrm{A}}^{2} \mathrm{~B}_{2} \times b_{1}\right)$. The overall qualitative agreement between the calculated 
vibronic spectrum and the experimental measurement confirms the general validity of our vibronic coupling approach, especially when compared to the Poisson spectrum where vibronic coupling is not treated (Figure 5(c)). However, some small discrepancies between the predicted and observed profiles still remain, indicating that a refinement to the present vibronic model might be needed.

\section{The $\tilde{D}^{2} B_{1}$ and $\tilde{E}^{2} B_{2}$ states}

The $\tilde{\mathrm{D}}^{2} \mathrm{~B}_{1}$ and $\tilde{\mathrm{E}}^{2} \mathrm{~B}_{2}$ states form a single photoelectron band with highly irregular vibrational structure which appears in the binding energy range $13.4-14.6 \mathrm{eV}$ (Figure 6(a)). The vertical separation of only $\sim 0.4 \mathrm{eV}$ (Table IV) between the $\tilde{\mathrm{D}}^{2} \mathrm{~B}_{1}$ and $\tilde{\mathrm{E}}^{2} \mathrm{~B}_{2}$ states suggests that these two states may couple vibronically via the two $a_{2}$ modes ( $v_{6}$ and $v_{7}$ ), $\left(\mathrm{B}_{1} \times \mathrm{B}_{2} \times a_{2} \supset \mathrm{A}_{1}\right)$. In the present work, an appropriate two-state LVC model was set up and its parameters were evaluated (Tables V and VII).

The intrastate coupling constants $\kappa$ (Table V) and the Poisson parameters (Eq. (9)), indicate that the most important totally symmetric modes for the $\tilde{\mathrm{D}}^{2} \mathrm{~B}_{1}$ and $\tilde{\mathrm{E}}^{2} \mathrm{~B}_{2}$ states are $v_{4}, v_{5}$ and $v_{2}$. The conical intersection between the potential energy surfaces associated with the $\tilde{\mathrm{D}}^{2} \mathrm{~B}_{1}$ and $\tilde{\mathrm{E}}^{2} \mathrm{~B}_{2}$ states occurs at $13.86 \mathrm{eV}$, which is almost at the adiabatic minimum $(13.84 \mathrm{eV})$ of the $\tilde{\mathrm{E}}^{2} \mathrm{~B}_{2}$ state. This can also be seen from the potential energy curves of the $\tilde{\mathrm{D}}^{2} \mathrm{~B}_{1}$ and $\tilde{\mathrm{E}}^{2} \mathrm{~B}_{2}$ states along $Q_{4}$, which is the most active coordinate for this pair of states (Figure S1 of the Supplementary Material). According to the interstate coupling constants $\lambda$ (Table VII), the coupling between the two states, via the $v_{6}\left(a_{2}\right)$ and $v_{7}\left(a_{2}\right)$ modes, is rather strong. As shown by the $\left|\lambda_{i j}^{s} / \omega_{s}\right|$ values (1.21 and 0.92, respectively), the coupling strength between the $\tilde{\mathrm{D}}^{2} \mathrm{~B}_{1}$ and $\tilde{\mathrm{E}}^{2} \mathrm{~B}_{2}$ states is much larger than that between the $\tilde{\mathrm{A}}^{2} \mathrm{~B}_{2}-\tilde{\mathrm{B}}^{2} \mathrm{~A}_{1}-\tilde{\mathrm{C}}^{2} \mathrm{~A}_{2}$ states. As a result of the stronger coupling, a double-well shape is predicted for the lower $\tilde{\mathrm{D}}^{2} \mathrm{~B}_{1}$ state potential energy surface with a stabilization energy of $0.07 \mathrm{eV}$. The adiabatic minimum of the $\tilde{\mathrm{D}}^{2} \mathrm{~B}_{1}$ state, corresponding to the symmetric $\left(\mathrm{C}_{2 \mathrm{v}}\right)$ molecular structure, at 
$13.69 \mathrm{eV}$ is therefore a transition state separating two equivalent molecular configurations of lower $\left(\mathrm{C}_{2}\right)$ symmetry. These two configurations are the true - though very shallow - minima with transition energies of $13.62 \mathrm{eV}$. Very complex non-adiabatic nuclear dynamics are therefore expected on the coupled $\tilde{\mathrm{D}}^{2} \mathrm{~B}_{1}$ and $\tilde{\mathrm{E}}^{2} \mathrm{~B}_{2}$ state potential energy surfaces. The calculated vibronic spectrum (Figure 6(b)) reflects this situation. In calculating the spectrum, all five $a_{1}$ modes $\left(v_{5}, v_{4}, v_{3}, v_{2}, v_{1}\right)$ were taken into account together with the two $a_{2}$ coupling modes $\left(v_{7}\right.$ and $\left.v_{6}\right)$. The corresponding harmonic oscillator basis set used in the calculations included functions with quantum numbers $n_{5}, n_{4}, n_{3}, n_{2}, n_{1}, n_{7}, n_{6}$ up to $10,20,5,10,5,30,30$, respectively.

As can be seen from the Poisson spectrum (Figure 6(c)), the vibrational progressions associated with the $\tilde{\mathrm{D}}^{2} \mathrm{~B}_{1}$ and $\tilde{\mathrm{E}}^{2} \mathrm{~B}_{2}$ states already overlap strongly at the non-interacting level and give rise to a single band with a highly complex envelope. The vibronic interaction further complicates the spectrum, giving rise to an envelope with erratic structure (Figure 6(b)). The agreement with the experimental spectrum (Figure 6(a)) improves but is only qualitative since the observed structure is only approximately reproduced by the vibronic calculations. However, in contrast to the Poisson spectrum, there are some features which are reproduced in a semi-quantitative manner. These are the nearly correct onset of the $\tilde{\mathrm{D}}^{2} \mathrm{~B}_{1}$ $\tilde{\mathrm{E}}^{2} \mathrm{~B}_{2}$ state band, and the overall width.

\section{THE INFLUENCE OF VIBRONIC COUPLING ON THE PHOTOELECTRON ANGULAR DISTRIBUTIONS}

Figure 7 shows the $\tilde{\mathrm{A}}^{2} \mathrm{~B}_{2}$ and $\tilde{\mathrm{B}}^{2} \mathrm{~A}_{1}$ state photoelectron bands, recorded at a photon energy of $20.5 \mathrm{eV}$, together with the corresponding photoelectron anisotropy parameters plotted as a function of binding energy. The $\beta$-independent photoelectron spectrum, and the $\beta$ values, were obtained using the procedure described by Powis et al. ${ }^{65}$ It is noticeable that the $\beta$-parameter associated with the $\tilde{\mathrm{A}}^{2} \mathrm{~B}_{2}$ state is lower in the low binding energy portion of the photoelectron band than it is in the high energy portion, particularly around $11.8 \mathrm{eV}$. Our vibronic calculations show that the adiabatic approximation is valid for the low energy 
portion of the $\tilde{\mathrm{A}}^{2} \mathrm{~B}_{2}$ state but that the peak in the photoelectron band around $11.8 \mathrm{eV}$ is due to coupling with the $\tilde{\mathrm{B}}^{2} \mathrm{~A}_{1}$ state. Figure 5(b) shows that the photoelectron intensity observed around $11.8 \mathrm{eV}$ is derived not only from the $\tilde{\mathrm{A}}^{2} \mathrm{~B}_{2}$ state (green bars), but also from the $\tilde{\mathrm{B}}^{2} \mathrm{~A}_{1}$ state (red bars), through vibronic coupling. The intensity associated with the two strong peaks at binding energies of $\sim 11.85$ and $11.95 \mathrm{eV}$ originates mainly from the $\tilde{\mathrm{B}}^{2} \mathrm{~A}_{1}$ state. In the binding energy regions coinciding with these peaks, the value of the $\beta$-parameter is $\sim 0.55$, whereas in the low energy portion of the $\tilde{\mathrm{A}}^{2} \mathrm{~B}_{2}$ state band a significantly lower value is observed. The high value of the anisotropy parameter around $11.8 \mathrm{eV}$ is due to vibronic coupling between the $\tilde{\mathrm{A}}^{2} \mathrm{~B}_{2}$ and $\tilde{\mathrm{B}}^{2} \mathrm{~A}_{1}$ states. This coupling not only enhances the photoelectron intensity, but also results in the $\beta$-parameter for the notionally high energy portion of the $\tilde{\mathrm{A}}^{2} \mathrm{~B}_{2}$ state band having a value similar to that in the peak around $11.95 \mathrm{eV}$, whose intensity derives almost exclusively from the $\tilde{\mathrm{B}}^{2} \mathrm{~A}_{1}$ state.

We have measured photoelectron spectra, and derived the corresponding $\beta$-parameters, in the photon energy range $19-90 \mathrm{eV}{ }^{68}$ These measurements show that the value of the photoelectron anisotropy parameter for the peak occurring around $11.8 \mathrm{eV}$ is always similar to those corresponding to the two main components of the $\tilde{\mathrm{B}}^{2} \mathrm{~A}_{1}$ state band, rather than the value characterizing the low energy portion of the $\tilde{\mathrm{A}}^{2} \mathrm{~B}_{2}$ state band. This behavior is particularly evident in the excitation range around $40 \mathrm{eV}$, due to the influence of the $\mathrm{Cl} 3 \mathrm{p}$ Cooper minimum. ${ }^{73}$

The effect of vibronic interactions on the electronic state photoelectron angular distributions and branching ratios of dichloroethene is considered in detail by Powis et al. ${ }^{68}$

\section{SUMMARY}

The ADC(3) approach has been employed to calculate the complete valence shell ionization spectrum of dichloroethene. In addition, vertical ionization energies have been computed using the OVGF and the EOM-IP-CCSD methods. The theoretical results agree well with each other and with the measurements, thereby allowing assignments to be 
proposed for most of the structure observed in the experimental spectra, including the innervalence regions dominated by satellite states.

The vibrational structure occurring in the lowest photoelectron bands has been studied using the LVC formalism for model Hamiltonians in the diabatic electronic basis. ${ }^{6,34}$ The LVC models were parameterized using data from the OVGF and ground state MP2 calculations.

While the adiabatic approximation holds for the $\tilde{\mathrm{X}}^{2} \mathrm{~B}_{1}$ state band, the next group of states, $\tilde{\mathrm{A}}^{2} \mathrm{~B}_{2}, \tilde{\mathrm{B}}^{2} \mathrm{~A}_{1}$ and $\tilde{\mathrm{C}}^{2} \mathrm{~A}_{2}$, form a multistate vibronic coupling problem, and give rise to a complex photoelectron band system. The experimental spectrum exhibits vibrational structure only in the low energy portion of the $\tilde{\mathrm{A}}{ }^{2} \mathrm{~B}_{2}$ state band. The remainder of the spectrum is rather diffuse and structureless. The most striking feature is the splitting of the $\tilde{\mathrm{B}}^{2} \mathrm{~A}_{1}$ state band into two strong components. This cannot be explained in terms of noninteracting cationic states.

In the first stage of our study, the influence of the other cationic states was excluded by computing the vertical ionization spectra using the OVGF and the ADC(3) propagator methods. A closer examination of the potential energy surfaces associated with the $\tilde{\mathrm{A}}^{2} \mathrm{~B}_{2}$, $\tilde{\mathrm{B}}^{2} \mathrm{~A}_{1}$ and $\tilde{\mathrm{C}}^{2} \mathrm{~A}_{2}$ states revealed a possibility of vibronic coupling among these states. Therefore, the LVC model, accounting for all three states, was set up and used to calculate the spectrum. Our results show that vibronic coupling gives rise to conical intersections between the corresponding potential energy surfaces near 11.87 and $12.39 \mathrm{eV}$. The $\tilde{\mathrm{B}}^{2} \mathrm{~A}_{1}$ and $\tilde{\mathrm{C}}^{2} \mathrm{~A}_{2}$ state bands therefore lie entirely within the domain of non-adiabatic nuclear dynamics, leading to highly complex structure in the computed vibronic spectrum. This theoretical spectrum is in good qualitative agreement with measurements, confirming the adequate level of the present vibronic treatment. In contrast, the Franck Condon calculations, using a model Hamiltonian without vibronic coupling, are unable to reproduce the spectrum even qualitatively. Further theoretical studies of the vibronic coupling between the $\tilde{\mathrm{A}}^{2} \mathrm{~B}_{2}, \tilde{\mathrm{B}}^{2} \mathrm{~A}_{1}$ and $\tilde{\mathrm{C}}^{2} \mathrm{~A}_{2}$ states are required to overcome the difficulties with the interstate coupling constants $\lambda$ within the LVC formalism. 
Our theoretical investigations show that the $\tilde{\mathrm{D}}^{2} \mathrm{~B}_{1}$ and $\tilde{\mathrm{E}}^{2} \mathrm{~B}_{2}$ states are also involved in strong vibronic interaction with each other. The conical intersection occurs at $13.86 \mathrm{eV}$, in the vicinity of the adiabatic minimum of the $\tilde{\mathrm{E}}^{2} \mathrm{~B}_{2}$ state. The single photoelectron band resulting from this interaction is characterized by highly irregular structure, reflecting the non-adiabatic nuclear dynamics occurring on the two coupled potential energy surfaces. The LVC model predicts a double-minimum potential energy surface for the $\tilde{\mathrm{D}}^{2} \mathrm{~B}_{1}$ state, characterized by a stabilization energy of $0.07 \mathrm{eV}$. This means that a molecular structure of lower $\left(\mathrm{C}_{2}\right)$ symmetry can be expected for the $\tilde{\mathrm{D}}^{2} \mathrm{~B}_{1}$ state. This suggestion, however, needs to be verified using more accurate electronic structure calculations since the presently predicted corresponding minima on the potential energy surface are very shallow.

Vibronic interactions have been shown to affect the photoionization dynamics, as characterized by the photoelectron anisotropy parameters. The $\beta$-values corresponding to the high binding energy region of the $\tilde{\mathrm{A}}^{2} \mathrm{~B}_{2}$, state band are similar to those of the neighbouring $\tilde{\mathrm{B}}^{2} \mathrm{~A}_{1}$ state band, to which it is vibronically coupled, rather than the $\beta$-parameters determined for the low energy portion of the $\tilde{\mathrm{A}}^{2} \mathrm{~B}_{2}$ state band. This coupling also modifies the photoelectron band shapes from those predicted through Franck-Condon simulations.

\section{SUPPLEMENTARY MATERIAL}

See supplementary material for seam minima and representative potential energy curves along $Q_{4}$ and $Q_{5}$ of some low-energy cationic states of dichloroethene, and potential energy curves of the neutral ground state and two low-lying cationic states along the nontotally symmetric $\left(b_{2}\right)$ coordinates.

\section{ACKNOWLEDGMENTS}

A.B.T. and A.D.S. gratefully acknowledge the grant, Grant No. 4.1671.2017/4.6, from the Ministry of Education and Science of the Russian Federation. D.M.P.H. is grateful to the Science and Technology Facilities Council (United Kingdom) for financial support. We thank 
the Synchrotron SOLEIL staff for running the facility and providing PLÉIADES beamtime under Project No. 20131329. 


\section{REFERENCES}

[1] M. Born and R. Oppenheimer, Ann. Phys. 84, 457 (1927).

[2] D. W. Turner, C. Baker, A. D. Baker, and C. R. Brundle, Molecular Photoelectron Spectroscopy (Wiley-Interscience, London, 1970).

[3] J. H. D. Eland, Photoelectron Spectroscopy (Butterworths, London, 1974).

[4] H. Siegbahn and L. Karlsson, in Handbuch der Physik, edited by W. Mehlhorn (Springer-Verlag, Berlin, 1982), Vol.31, p. 215.

[5] G. Herzberg, Molecular Spectra and Molecular Structure III: Electronic Spectra and Electronic Structure of Polyatomic Molecules (Van Nostrand Reinhold, New York, 1966).

[6] H. Köppel, W. Domcke, and L.S. Cederbaum, Adv. Chem. Phys. 57, 59 (1984).

[7] D. R. Yarkony, Rev. Mod. Phys. 68, 985 (1996).

[8] A. B. Trofimov, H. Köppel, and J. Schirmer, J. Chem. Phys. 109, 1025 (1998).

[9] G. A. Worth and L. S. Cederbaum, Ann. Rev. Phys. Chem. 55, 127 (2004).

[10] W. Domcke, D. R. Yarkony, and H. Köppel, eds., Conical Intersections: Electronic Structure, Dynamics and Spectroscopy (World Scientific, Singapore, 2004).

[11] S. Faraji, H.-D. Meyer, and H. Köppel, J. Chem. Phys. 129, 074311 (2008).

[12] R. Sarkar, S. R. Reddy, S. Mahapatra, and H. Köppel, Chem. Phys. 482, 39 (2017).

[13] L.S. Cederbaum, W. Domcke, H. Köppel, and W. von Niessen, Chem. Phys. 26, 169 (1977).

[14] H. Köppel, W. Domcke and L. S. Cederbaum, The multi-mode vibronic-coupling approach, in Conical Intersections, edited by W. Domcke, D. R. Yarkony and H. Köppel (World Scientific, New Jersey, 2004), p. 323.

[15] W. von Niessen, J. Schirmer, and L.S. Cederbaum, Comp. Phys. Reports 1, 57 (1984).

[16] V. G. Zakrzewski and J. V. Ortiz, Int. J. Quantum Chem. Suppl. 28, 23 (1994).

[17] J. V. Ortiz, in Computational Chemistry: Reviews of Current Trends, edited by J. Leszcynski (World Scientific, Singapore, 1997), Vol. 2, p. 1.

[18] E. Haller, H. Köppel, L. S. Cederbaum, G. Bieri, and W. von Niessen, Chem. Phys. Lett. 85, 12 (1982). 
[19] E. Haller, H. Köppel, L. S. Cederbaum, W. von Niessen, and G. Bieri, J. Chem. Phys. 78, 1359 (1983).

[20] D. M. P. Holland, M. A. MacDonald, M. A. Hayes, L. Karlsson, and B. Wannberg, Chem. Phys. 226, 351 (1998).

[21] N. Jonathan, K. Ross, and V. Tomlinson, Int. J. Mass Spectrom. Ion Phys. 4, 51 (1970).

[22] R. F. Lake and H. Thompson, Proc. Roy. Soc. Lond. A 315, 323 (1970.

[23] K. Wittel and H. Bock, Chem. Rev. 107, 317 (1974).

[24] K. Kimura, S. Katsumata, Y. Achiba, T. Yamazaki, and S. Iwata, Handbook of HeI Photoelectron Spectra of Fundamental Organic Molecules (Japan Scientific Societies Press, Tokyo, 1981).

[25] W. von Niessen, L. Åsbrink, and G. Bieri, J. Electron Spectrosc. Relat. Phenom. 26, 173 (1982).

[26] A. Berndtsson, E. Basilier, U. Gelius, J. Hedman, M. Klasson, R. Nilsson, C. Nordling, and S. Svensson, Phys. Scripta 12, 235 (1975).

[27] J. F. Ying and K. T. Leung, J. Electron Spectrosc. Relat. Phenom. 63, 75 (1993).

[28] M. A. Parkes, S. Ali, C. R. Howles, R. P. Tuckett, and A. E. R. Malins, Mol. Phys. 105, 907 (2007).

[29] Y. J. Bae and M. S. Kim, Int. J. Mass Spectrom. 267, 89 (2007).

[30] K.-C. Lau, H. K. Woo, P. Wang, X. Xing, and C. Y. Ng, J. Chem. Phys. 124, 224311 (2006).

[31] L. Mei, M. Chuaqui, C. P. Mathers, J. F. Ying, and K. T. Leung, J. Chem. Phys. 101, 2558 (1994).

[32] V. Galasso, J. Mol. Struct. (Theochem), 337, 249 (1995).

[33] K. Takeshita, J. Chem. Phys. 110, 6792 (1999).

[34] T. Pacher, L.S. Cederbaum, and H. Köppel, Adv. Chem. Phys. 84, 293 (1993).

[35] E.B. Wilson, Jr., J.C. Decius, and P.C. Cross, Molecular Vibrations (McGraw-Hill, New York 1955). 
[36] J. Cullum and R. Willoughby, Lanczos Algorithms for Large Symmetric Eigenvalue Problems (Birkhäuser, Boston, 1985), Vols. I and II.

[37] The general multistate vibronic coupling code written by A. B. Trofimov, Irkutsk State University.

[38] T. H. Dunning, J. Chem. Phys. 90, 1007 (1989).

[39] D.E. Woon and T.H. Dunning, J. Chem. Phys. 98, 1358 (1993).

[40] K. Kuchitsu, ed., Landolt-Bornstein: Group II: Atomic and Molecular Physics Volume 21: Structure Data of Free Polyatomic Molecules (Springer-Verlag, Berlin, 1992).

[41] G. Herzberg, Molecular Spectra and Molecular Structure II: Infrared and Raman Spectra of Polyatomic Molecules (van Nostrand Reinhold, New York, 1945).

[42] T. Shimanouchi, Tables of Molecular Vibrational Frequencies, Consolidated Volume I, (National Bureau of Standards, 1972).

[43] K. S. Pitzer and J. L. Hollenberg, J. Am. Chem. Soc. 76, 1493 (1954).

[44] M.J. Frisch et al., Gaussian 09, Revision C.01, Gaussian Inc. Wallingford, CT, 2010.

[45] L. S. Cederbaum, W. Domcke, J. Schirmer, and W. von Niessen, Adv. Chem. Phys. 65, 115 (1986).

[46] J. Schirmer, L. S. Cederbaum, and O. Walter, Phys. Rev. A 28, 1237 (1983).

[47] J. Schirmer and G. Angonoa, J. Chem. Phys. 91, 1754 (1989).

[48] A. B. Trofimov, J. Schirmer, D. M. P. Holland, L. Karlsson, R. Maripuu, K. Siegbahn, and A. W. Potts, Chem. Phys. 263, 167 (2001).

[49] A. W. Potts, A. B. Trofimov, J. Schirmer, D. M. P. Holland, and L. Karlsson, Chem. Phys. 271, 337 (2001).

[50] A. B. Trofimov, J. Schirmer, D. M. P. Holland, A. W. Potts, L. Karlsson, R. Maripuu, and K. Siegbahn, J. Phys. B: At., Mol. Opt. Phys. 35, 5051 (2002).

[51] D. M. P. Holland, A. W. Potts, L. Karlsson, I. L. Zaytseva, A. B. Trofimov, and J. Schirmer, Chem. Phys. 352, 205 (2008).

[52] A. W. Potts, D. M. P. Holland, I. Powis, L. Karlsson, A. B. Trofimov, and I. L. Bodzuk, Chem. Phys. 415, 84 (2013). 
[53] I. Powis, A. B. Trofimov, I. L. Bodzuk, D. M. P. Holland, A. W. Potts, and L. Karlsson, Chem. Phys. 415, 291 (2013).

[54] D. M. P. Holland, I. Powis, A. B. Trofimov, I. L. Bodzuk, D. Yu. Soshnikov, A. W. Potts, and L. Karlsson, Chem. Phys. 448, 61 (2015).

[55] H. Sekino and R.J. Bartlett, Int. J. Quantum Chem. Suppl. 18, 255 (1984).

[56] A. I. Krylov, Annu. Rev. Phys. Chem. 59, 433 (2008).

[57] M. Nooijen and J. G. Snijders, Int. J. Quantum Chem. Suppl. 26, 55 (1992).

[58] J. F. Stanton and J. Gauss, J. Chem. Phys. 103, 1064 (1995).

[59] Y. Shao et al., Mol. Phys. 113, 184 (2015).

[60] The ADC(3) code originally written by G. Angonoa, O. Walter, and J. Schirmer; Further developed by M. K. Scheller and A. B. Trofimov.

[61] M. W. Schmidt, K. K. Baldridge, J. A. Boatz, S. T. Elbert, M. S. Gordon, J. H. Jensen, S. Koseki, N. Matsunaga, K. A. Nguyen, S. Su, T. L. Windus, M. Dupuis, and J. A. Montgomery, J. Comput. Chem. 14, 1347 (1993).

[62] M. S. Gordon and M. W. Schmidt, in Advances in Electronic Structure Theory: GAMESS a Decade Later, edited by C. E. Dykstra, G. Frenking, K. S. Kim, and G. E. Scuseria (Elsevier, Amsterdam, 2005), p. 1167.

[63] G. Schaftenaar and J. H. Noordik, "Molden: A pre- and post-processing program for molecular and electronic structures", J. Comput.-Aided Mol. Des. 14, 123 (2000).

[64] J. Söderström, A. Lindblad, A. N. Grum-Grzhimailo, O. Travnikova, C. Nicolas, S. Svensson, and C. Miron, New J. Phys. 13, 073014 (2011).

[65] I. Powis, D. M. P. Holland, E. Antonsson, M. Patanen, C. Nicolas, C. Miron, M. Scheider, D. Yu. Soshnikov, A. Dreuw, and A. B. Trofimov, J. Chem. Phys. 143, 144304 (2015).

[66] B. Lagarde, F. Sirotti, A. Taleb-Ibrahimi, C. Miron, and F. Polack, J. Phys. Conf. Ser. 425, 152022 (2013).

[67] J. Jauhiainen, A. Ausmees, A. Kivimäki, S. J. Osborne, A. Naves de Brito, S. Aksela, S. Svensson, and H. Aksela, J. Electron Spectrosc. Relat. Phenom. 69, 181 (1994). 
[68] I. Powis, R. C. Menzies, D. M. P. Holland, A. B. Trofimov, A. D. Skitnevskaya, E. V. Gromov, E. Antonsson, M. Patanen, C. Nicolas, and C. Miron, in preparation.

[69] R. S. Mulliken, J. Chem. Phys. 23, 1833 (1955).

[70] F. Santoro, R. Improta, A. Lami, J. Bloino, and V. Barone, J. Chem. Phys. 126, 084509 (2007).

[71] F. Santoro, A. Lami, R. Improta, J. Bloino, and V. Barone, J. Chem. Phys. 128, 224311 (2008).

[72] F. Duschinsky, Acta Physicochim. U. R. S. S. 1, 551 (1937).

[73] S. T. Manson, A. Msezane, A. F. Starace, and S. Shahabi, Phys. Rev. A 20, 1005 (1979). 
TABLE I. Calculated (MP2/cc-pVTZ) and experimental equilibrium geometrical parameters of dichloroethene (bond lengths in $\AA$ and angles in degrees) in the neutral ground state $\left(\tilde{X}^{1} A_{1}\right)$ and lowest cationic state $\left(\mathrm{X}^{2} \mathrm{~B}_{1}\right)$.

\begin{tabular}{|c|c|c|c|}
\hline \multirow{2}{*}{ Parameter } & \multicolumn{2}{|c|}{ MP2, this work } & Experiment $^{\mathrm{a}}$ \\
\cline { 2 - 4 } & $\tilde{\mathrm{X}}{ }^{1} \mathrm{~A}_{1}$ & $\tilde{\mathrm{X}}^{2} \mathrm{~B}_{1}$ & $\tilde{\mathrm{X}}^{1} \mathrm{~A}_{1}$ \\
\hline Bond length & & & \\
\hline $\mathrm{C}=\mathrm{C}$ & 1.333 & 1.405 & 1.317 \\
\hline $\mathrm{C}-\mathrm{H}$ & 1.079 & 1.083 & 1.101 \\
\hline $\mathrm{C}-\mathrm{Cl}$ & 1.713 & 1.634 & 1.717 \\
\hline Angle & & & \\
\hline $\mathrm{C}=\mathrm{C}-\mathrm{H}$ & 120.18 & 119.03 & 123.18 \\
\hline $\mathrm{C}=\mathrm{C}-\mathrm{Cl}$ & 124.48 & 123.46 & 124.22 \\
\hline \hline
\end{tabular}

${ }^{a}$ Kuchitsu $^{40}$ 
TABLE II. Calculated (MP2/cc-pVTZ) and observed frequencies $\left(\mathrm{cm}^{-1}\right)$ of dichloroethene in the neutral ground state $\left(\tilde{X}^{1} A_{1}\right)$ and the lowest cationic state $\left(\mathrm{X}^{2} \mathrm{~B}_{1}\right)$.

\begin{tabular}{|c|c|c|c|c|}
\hline \multirow{2}{*}{ Mode $^{\mathrm{a}}$} & \multirow{2}{*}{ Type $^{\mathrm{b}}$} & \multicolumn{2}{|c|}{ MP2, this work } & Experiment $^{\mathrm{c}}$ \\
\cline { 3 - 5 } & & $\tilde{\mathrm{X}}^{1} \mathrm{~A}_{1}$ & $\tilde{\mathrm{X}}^{2} \mathrm{~B}_{1}$ & $\tilde{\mathrm{X}}^{1} \mathrm{~A}_{1}$ \\
\hline$a_{1}$ modes & & & \\
\hline$v_{1}$ & $\mathrm{CH}$ stretch & 3266 & 3233 & 3077 \\
\hline$v_{2}$ & $\mathrm{CC}$ stretch & 1641 & 1477 & 1587 \\
\hline$v_{3}$ & $\mathrm{CH}$ bend & 1213 & 1241 & 1179 \\
\hline$v_{4}$ & $\mathrm{CCl}$ stretch & 745 & 849 & 711 \\
\hline$v_{5}$ & $\mathrm{CCCl}$ deform & 168 & 185 & 173 \\
\hline$a_{2}$ modes & & & & \\
\hline$v_{6}$ & $\mathrm{CH}$ bend & 901 & 940 & 876 \\
\hline$v_{7}$ & torsion & 419 & 293 & 406 \\
\hline$b_{1}$ modes & & & & \\
\hline$v_{8}$ & $\mathrm{CH}$ bend & 720 & 762 & 697 \\
\hline$b_{2}$ modes & & & & \\
\hline$v_{9}$ & $\mathrm{CH}$ stretch & 3244 & 3219 & 3072 \\
\hline$v_{10}$ & $\mathrm{CH}$ bend & 1327 & 1396 & 1303 \\
\hline$v_{11}$ & $\mathrm{CCl}$ stretch & 883 & 1021 & 857 \\
\hline$v_{12}$ & $\mathrm{CCCl}$ deform & 581 & 594 & 571 \\
\hline \hline
\end{tabular}

${ }^{a}$ Normal modes are numbered according to Herzberg. ${ }^{41}$

b Normal mode descriptions from Shimanouchi, ${ }^{42}$ and confirmed by the present calculations.

${ }^{c}$ Refs. 42 and 43. 
Table III. Mulliken atomic population in the outer valence molecular orbitals of dichloroethene (units are electrons; sum over all atoms is 2) calculated at the HF/cc-pVTZ level.

\begin{tabular}{|c|c|c|c|c|c|c|c|c|}
\hline Atom & $\begin{array}{c}3 \mathrm{~b}_{1} \\
(\pi)\end{array}$ & $\begin{array}{c}9 \mathrm{~b}_{2} \\
\left(\sigma_{\mathrm{ClLP}}\right)\end{array}$ & $\begin{array}{c}10 \mathrm{a}_{1} \\
\left(\sigma_{\mathrm{Cl} \mathrm{LP}}\right)\end{array}$ & $\begin{array}{c}2 \mathrm{a}_{2} \\
\left(\pi_{\mathrm{ClLP}}\right)\end{array}$ & $\begin{array}{c}2 \mathrm{~b}_{1} \\
\left(\pi_{\mathrm{ClLP}}\right)\end{array}$ & $\begin{array}{c}8 \mathrm{~b}_{2} \\
(\sigma)\end{array}$ & $\begin{array}{c}9 \mathrm{a}_{1} \\
(\sigma)\end{array}$ & $\begin{array}{c}8 \mathrm{a}_{1} \\
(\sigma)\end{array}$ \\
\hline $\mathrm{C}$ & 0.58 & 0.04 & 0.08 & 0.06 & 0.40 & 0.33 & 0.39 & 0.59 \\
\hline $\mathrm{H}$ & 0.01 & 0.02 & 0.05 & 0.0 & 0.0 & 0.15 & 0.0 & 0.28 \\
\hline $\mathrm{Cl}$ & 0.41 & 0.94 & 0.87 & 0.94 & 0.59 & 0.52 & 0.61 & 0.13 \\
\hline \hline
\end{tabular}


Table IV. Energies $E(\mathrm{eV})$ and intensities $P$ of the outer valence vertical ionization transitions in dichloroethene computed using the HF, OVGF, ADC(3), and EOM-IP-CCSD methods, and the cc-pVTZ basis set. The experimental values are given for comparison.

\begin{tabular}{|c|c|c|c|c|c|c|c|c|c|}
\hline \multirow{2}{*}{$\begin{array}{c}\text { Cationic } \\
\text { state }\end{array}$} & \multirow{2}{*}{$\begin{array}{c}\text { Molecular } \\
\text { orbital }\end{array}$} & \multirow{2}{*}{ Type } & \multirow{2}{*}{$\frac{\mathrm{HF}}{E}$} & \multicolumn{2}{|c|}{ OVGF } & \multicolumn{2}{|c|}{$\mathrm{ADC}(3)$} & \multirow{2}{*}{$\begin{array}{c}\text { EOM-IP- } \\
\text { CCSD }\end{array}$} & \multirow{2}{*}{ Expt. $^{a}$} \\
\hline & & & & $E$ & $P$ & $E$ & $P$ & & \\
\hline$\tilde{\mathrm{X}}^{2} \mathrm{~B}_{1}$ & $3 b_{1}$ & $\pi$ & 9.93 & 9.70 & 0.91 & 9.75 & 0.90 & 9.78 & $9.8^{\mathrm{b}}$ \\
\hline$\tilde{\mathrm{A}}^{2} \mathrm{~B}_{2}$ & $9 b_{2}$ & $\sigma_{\mathrm{Cl} L P}$ & 12.42 & 11.68 & 0.90 & 11.64 & 0.89 & 11.66 & 11.70 \\
\hline$\tilde{\mathrm{B}}^{2} \mathrm{~A}_{1}$ & $10 \mathrm{a}_{1}$ & $\sigma_{\mathrm{Cl} L P}$ & 12.82 & 11.98 & 0.90 & 11.98 & 0.89 & 12.00 & 12.06 \\
\hline$\tilde{\mathrm{C}}^{2} \mathrm{~A}_{2}$ & $2 \mathrm{a}_{2}$ & $\pi_{\mathrm{Cl} \mathrm{LP}}$ & 13.30 & 12.51 & 0.90 & 12.48 & 0.88 & 12.53 & 12.50 \\
\hline$\tilde{\mathrm{D}}^{2} \mathrm{~B}_{1}$ & $2 b_{1}$ & $\pi_{\mathrm{Cl} L \mathrm{P}}$ & 14.93 & 13.89 & 0.88 & 13.93 & 0.85 & 13.95 & 13.80 \\
\hline$\tilde{\mathrm{E}}^{2} \mathrm{~B}_{2}$ & $8 b_{2}$ & $\sigma$ & 15.07 & 14.10 & 0.90 & 14.21 & 0.89 & 14.20 & 14.21 \\
\hline${ }^{2} \mathrm{~A}_{2}$ & $2 \mathrm{a}_{2}$ & $2 h-1 p$ & & & & 14.58 & 0.01 & & \\
\hline${ }^{2} \mathrm{~A}_{1}$ & $9 \mathrm{a}_{1}$ & $2 h-1 p$ & & & & 15.68 & 0.27 & & \\
\hline$\tilde{\mathrm{F}}^{2} \mathrm{~A}_{1}$ & $9 a_{1}$ & $\sigma$ & 16.80 & 15.64 & 0.89 & 15.79 & 0.60 & 15.75 & 15.68 \\
\hline$\tilde{\mathrm{G}}^{2} \mathrm{~A}_{1}$ & $8 a_{1}$ & $\sigma$ & 19.01 & 17.21 & 0.87 & 17.38 & 0.81 & 17.31 & 16.9 \\
\hline
\end{tabular}

a Peak maxima, as estimated from the present photoelectron spectrum.

$\mathrm{b}$ The adiabatic transition energy is $9.659 \mathrm{eV}$. 
TABLE V. Intrastate coupling constants $\kappa(\mathrm{eV})$ for the six lowest states of the dichloroethene radical cation.

\begin{tabular}{|c|c|c|c|c|c|}
\hline \multirow{2}{*}{ State } & \multicolumn{5}{|c|}{ Totally symmetric $\left(a_{1}\right)$ vibrational modes } \\
\cline { 2 - 6 } & $v_{1}$ & $v_{2}$ & $v_{3}$ & $v_{4}$ & $v_{5}$ \\
\hline$\tilde{\mathrm{X}}{ }^{2} \mathrm{~B}_{1}$ & -0.006 & -0.195 & -0.049 & 0.079 & -0.007 \\
\hline$\tilde{\mathrm{A}}^{2} \mathrm{~B}_{2}$ & -0.029 & 0.039 & -0.028 & -0.019 & -0.069 \\
\hline$\tilde{\mathrm{B}}^{2} \mathrm{~A}_{1}$ & -0.034 & -0.059 & -0.015 & 0.036 & 0.047 \\
\hline$\tilde{\mathrm{C}}^{2} \mathrm{~A}_{2}$ & -0.004 & 0.049 & 0.003 & -0.078 & -0.032 \\
\hline$\tilde{\mathrm{D}}^{2} \mathrm{~B}_{1}$ & 0.002 & -0.046 & -0.002 & -0.127 & 0.016 \\
\hline$\tilde{\mathrm{E}}^{2} \mathrm{~B}_{2}$ & -0.149 & 0.150 & -0.034 & -0.088 & -0.003 \\
\hline \hline
\end{tabular}


TABLE VI. Calculated (OVGF/cc-pVTZ, LVC model) vertical $\left(E_{v}\right)$ and adiabatic $\left(E_{0-0}\right)$ energies for the six lowest ionization transitions in dichloroethene. $E_{\text {int }}$ is the minimal energy of the conical intersection between the potential energy surfaces for the two groups of cationic states, $\left(\tilde{\mathrm{A}}^{2} \mathrm{~B}_{2}\right.$, $\left.\tilde{B}^{2} \mathrm{~A}_{1}, \tilde{C}^{2} \mathrm{~A}_{2}\right)$ and $\left(\tilde{D}^{2} \mathrm{~B}_{1}, \tilde{E}^{2} \mathrm{~B}_{2}\right)$, treated in the present vibronic coupling models. All values are in $\mathrm{eV}$.

\begin{tabular}{|c|c|c|c|c|c|}
\hline \hline & & & \multicolumn{3}{|c|}{$E_{\text {int }}{ }^{\mathrm{a}}$} \\
\cline { 5 - 7 } State & $E_{v}$ & $E_{0-0}$ & $\tilde{\mathrm{A}}^{2} \mathrm{~B}_{2}$ & $\tilde{\mathrm{B}}^{2} \mathrm{~A}_{1}$ & $\tilde{\mathrm{E}}^{2} \mathrm{~B}_{2}$ \\
\hline$\tilde{\mathrm{X}}^{2} \mathrm{~B}_{1}$ & 9.70 & $9.43^{\mathrm{b}}$ & & & \\
\hline$\tilde{\mathrm{A}}^{2} \mathrm{~B}_{2}$ & 11.68 & 11.43 & & & \\
$\tilde{\mathrm{B}}^{2} \mathrm{~A}_{1}$ & 11.98 & 11.84 & 11.87 & & \\
\hline$\tilde{\mathrm{C}}^{2} \mathrm{~A}_{2}$ & 12.51 & 12.39 & 13.99 & 12.39 & \\
\hline$\tilde{\mathrm{D}}^{2} \mathrm{~B}_{1}$ & 13.89 & $13.69^{\mathrm{c}}$ & & & 13.86 \\
\hline$\tilde{\mathrm{E}}^{2} \mathrm{~B}_{2}$ & 14.10 & 13.84 & & & \\
\hline \hline
\end{tabular}

${ }^{a}$ Obtained from the respective LVC models for pairs of interacting states.

${ }^{\mathrm{b}}$ The present $\triangle \mathrm{MP} 2$ value is $9.64 \mathrm{eV}$ (obtained as the difference between the total energies, including electronic and zero-point vibrational energy components, of the $\tilde{\mathrm{X}}^{1} \mathrm{~A}_{1}$ and $\tilde{\mathrm{X}}^{2} \mathrm{~B}_{1}$ states, at their equilibrium geometries). The present experimental adiabatic energy is $9.66 \mathrm{eV}$.

${ }^{\mathrm{c}}$ Saddle point. The present LVC model predicts a double-minimum potential energy surface, characterized by a stabilization energy of $0.07 \mathrm{eV}$. 
TABLE VII. Interstate coupling constants $\lambda(\mathrm{eV})$ within the two groups of cationic states $\left(\tilde{\mathrm{A}}^{2} \mathrm{~B}_{2}, \tilde{\mathrm{B}}^{2} \mathrm{~A}_{1}\right.$, $\left.\tilde{\mathrm{C}}^{2} \mathrm{~A}_{2}\right)$ and $\left(\tilde{\mathrm{D}}^{2} \mathrm{~B}_{1}, \tilde{\mathrm{E}}^{2} \mathrm{~B}_{2}\right)$, treated in the present vibronic coupling models.

\begin{tabular}{|c|c|c|c|c|c|c|c|}
\hline \multirow{2}{*}{$\begin{array}{c}\text { Interacting } \\
\text { states }\end{array}$} & \multicolumn{7}{|c|}{ Coupling vibrational modes } \\
\hline & $v_{6}\left(a_{2}\right)$ & $v_{7}\left(a_{2}\right)$ & $v_{9}\left(b_{2}\right)$ & $v_{10}\left(b_{2}\right)$ & $v_{11}\left(b_{2}\right)$ & $v_{12}\left(b_{2}\right)$ & $v_{8}\left(b_{1}\right)$ \\
\hline$\tilde{\mathrm{A}}^{2} \mathrm{~B}_{2}-\tilde{\mathrm{B}}^{2} \mathrm{~A}_{1}$ & & & $0.012^{\mathrm{a}}$ & 0.066 & $0.029^{\mathrm{a}}$ & $0.002^{\mathrm{a}}$ & \\
\hline$\tilde{\mathrm{B}}^{2} \mathrm{~A}_{1}-\tilde{\mathrm{C}}^{2} \mathrm{~A}_{2}$ & 0.047 & 0.033 & & & & & \\
\hline$\tilde{\mathrm{A}}{ }^{2} \mathrm{~B}_{2}-\tilde{\mathrm{C}}^{2} \mathrm{~A}_{2}$ & & & & & & & 0.012 \\
\hline $\mathrm{D}^{2} \mathrm{~B}_{1}-\mathrm{E}^{2} \mathrm{~B}_{2}$ & 0.135 & 0.048 & & & & & \\
\hline
\end{tabular}

${ }^{a}$ An approximation accounting for only the lower $\tilde{\mathrm{A}}^{2} \mathrm{~B}_{2}$ surface has been used to determine the constant $\lambda$, since the potential energy surface of the upper $\tilde{\mathrm{B}}^{2} \mathrm{~A}_{1}$ state along this mode cannot be described by the present linear vibronic coupling model (see text for details). 
Figure captions

Figure 1. Schematic representation of dichloroethene showing the adopted axis orientation.

Figure 2. Plots of the eight highest occupied molecular orbitals of dichloroethene produced using the results of the HF/cc-pVTZ calculations.

Figure 3. The valence shell photoelectron spectrum of dichloroethene: (a) experimental spectrum recorded at a photon energy of $80 \mathrm{eV}$; (b) theoretical spectrum obtained using the ADC(3) method and the cc-pVTZ basis set.

Figure 4. The $\tilde{\mathrm{X}}^{2} \mathrm{~B}_{1}$ state photoelectron band: (a) experimental spectrum; (b) theoretical spectrum obtained using Franck-Condon simulations employing the adiabatic potential energy surfaces of the ground and cationic states computed at the MP2/cc-pVTZ level of theory; (c) theoretical spectrum obtained using the LVC model (Poisson spectrum) based on the parameters from the OVGF/cc-pVTZ calculations (see text for details).

Figure 5. The $\tilde{\mathrm{A}}^{2} \mathrm{~B}_{2}, \quad \tilde{\mathrm{B}}^{2} \mathrm{~A}_{1}$ and $\tilde{\mathrm{C}}^{2} \mathrm{~A}_{2}$ state photoelectron bands: (a) experimental spectrum; (b) theoretical spectrum taking into account the vibronic coupling between the three states obtained using the LVC model based on the parameters from the OVGF/cc-pVTZ calculations; (c) theoretical spectrum obtained using the same model as in (b), but without the vibronic coupling. This is equivalent to the Poisson spectra for the three states (see text for details). Individual transitions to vibronic states of $\mathrm{B}_{2}, \mathrm{~A}_{1}$, and $\mathrm{A}_{2}$ symmetry are shown in the spectra as green, red, and violet bars, respectively. For improved clarity the intensities of the bars representing the vibronic states of $\mathrm{B}_{2}, \mathrm{~A}_{1}$ and $\mathrm{A}_{2}$ symmetry were scaled by factors of $0.6,1.0$, and 4 , respectively, in (b), and by $0.6,0.6$, and 0.6 , respectively, in (c). 
Figure 6. The $\tilde{\mathrm{D}}^{2} \mathrm{~B}_{1}$ and $\tilde{\mathrm{E}}^{2} \mathrm{~B}$ state photoelectron band system: (a) experimental spectrum; (b) theoretical spectrum taking into account the vibronic coupling between the two states obtained using the LVC model based on the parameters from the OVGF/cc-pVTZ calculations; (c) theoretical spectrum obtained using the same model as in (b), but without the vibronic coupling. This is equivalent to the Poisson spectra for the two states (see text for details). Individual transitions to vibronic states of $\mathrm{B}_{1}$ and $\mathrm{B}_{2}$ symmetry are shown in the spectra as red and violet bars, respectively. For improved clarity the intensities of the bar spectra were scaled by a factors 0.6 and 1.0, respectively, in (b), and by 0.6 and 0.6 , respectively, in (c).

Figure 7. The $\tilde{\mathrm{A}}^{2} \mathrm{~B}_{2}$ and $\tilde{\mathrm{B}}^{2} \mathrm{~A}_{1}$ state photoelectron bands (blue), recorded at a photon energy of $20.5 \mathrm{eV}$, together with the corresponding photoelectron anisotropy parameters (red). 


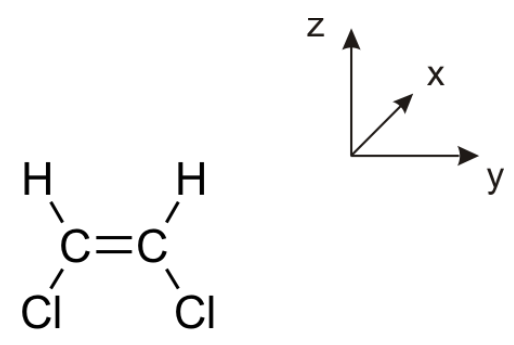

Figure 1 


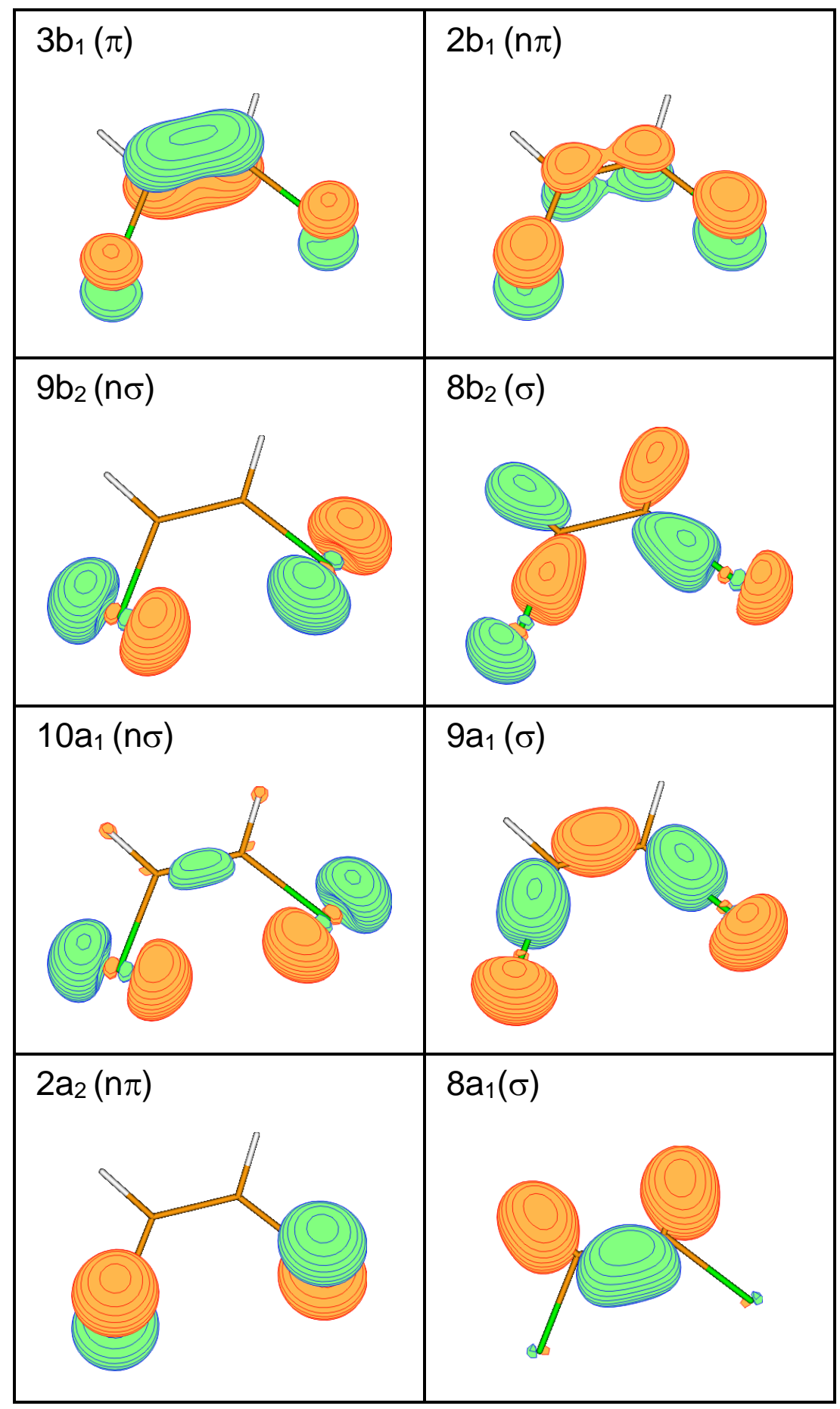

Figure 2 


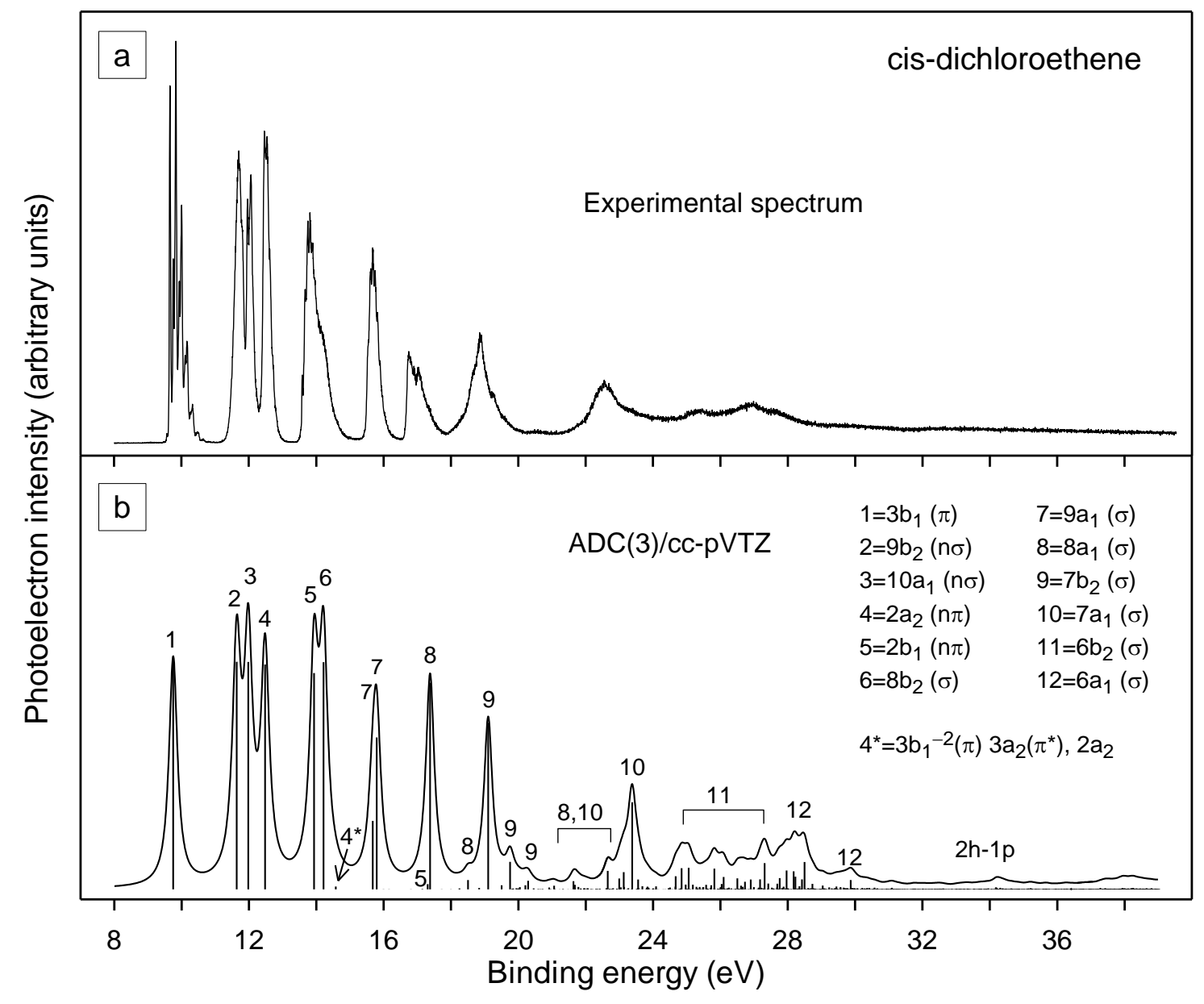

Figure 3 


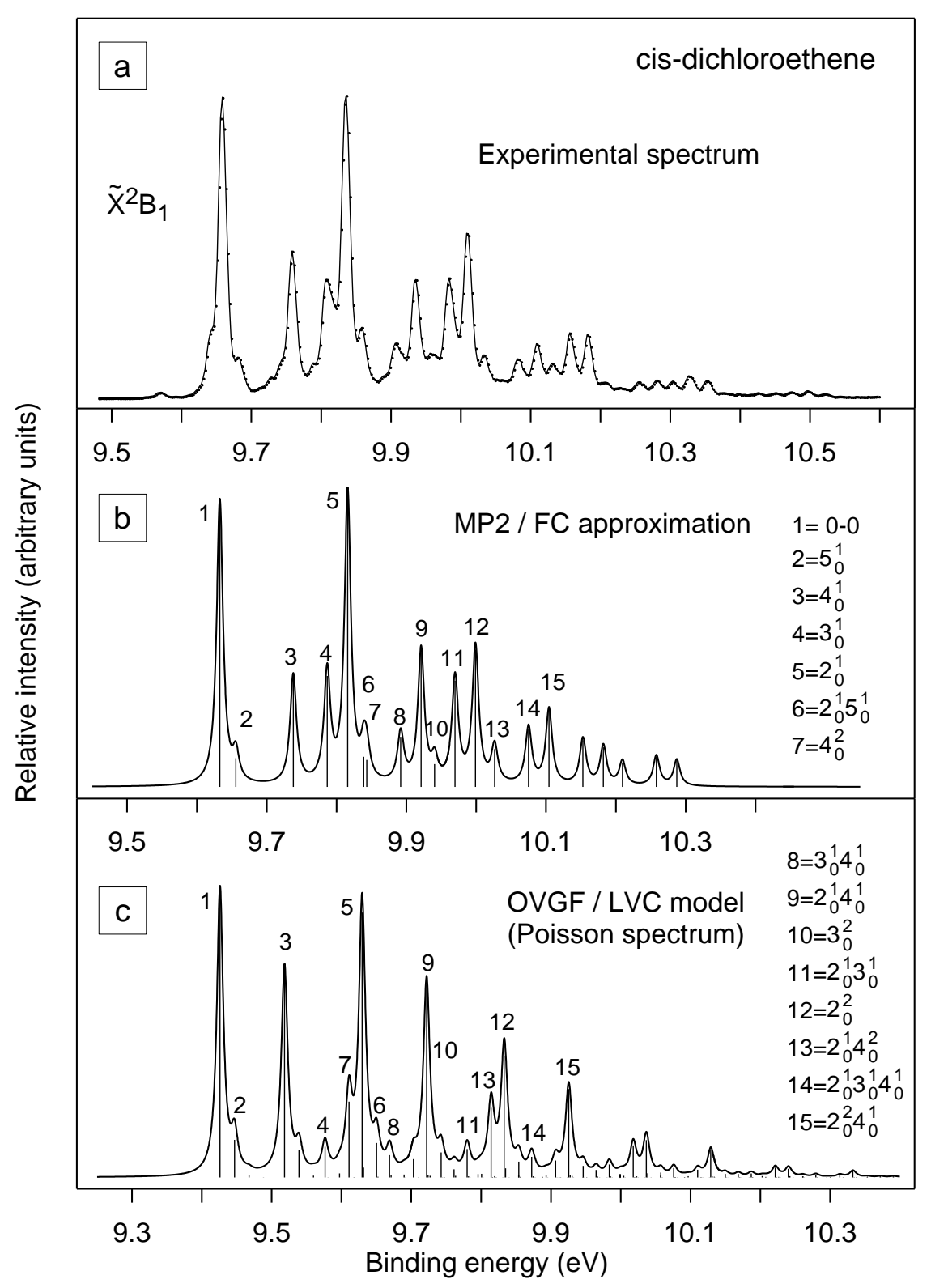

Figure 4 


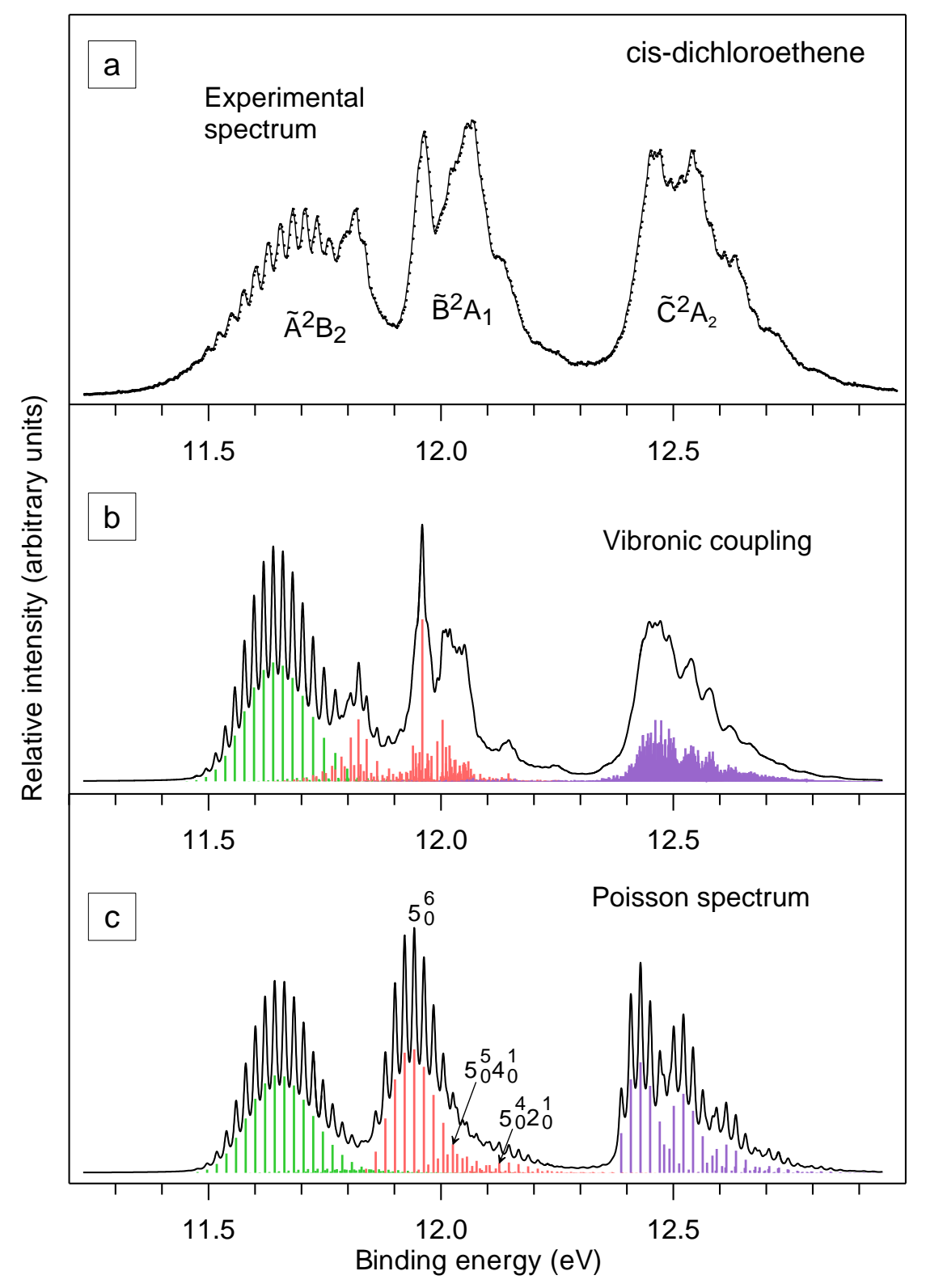

Figure 5 


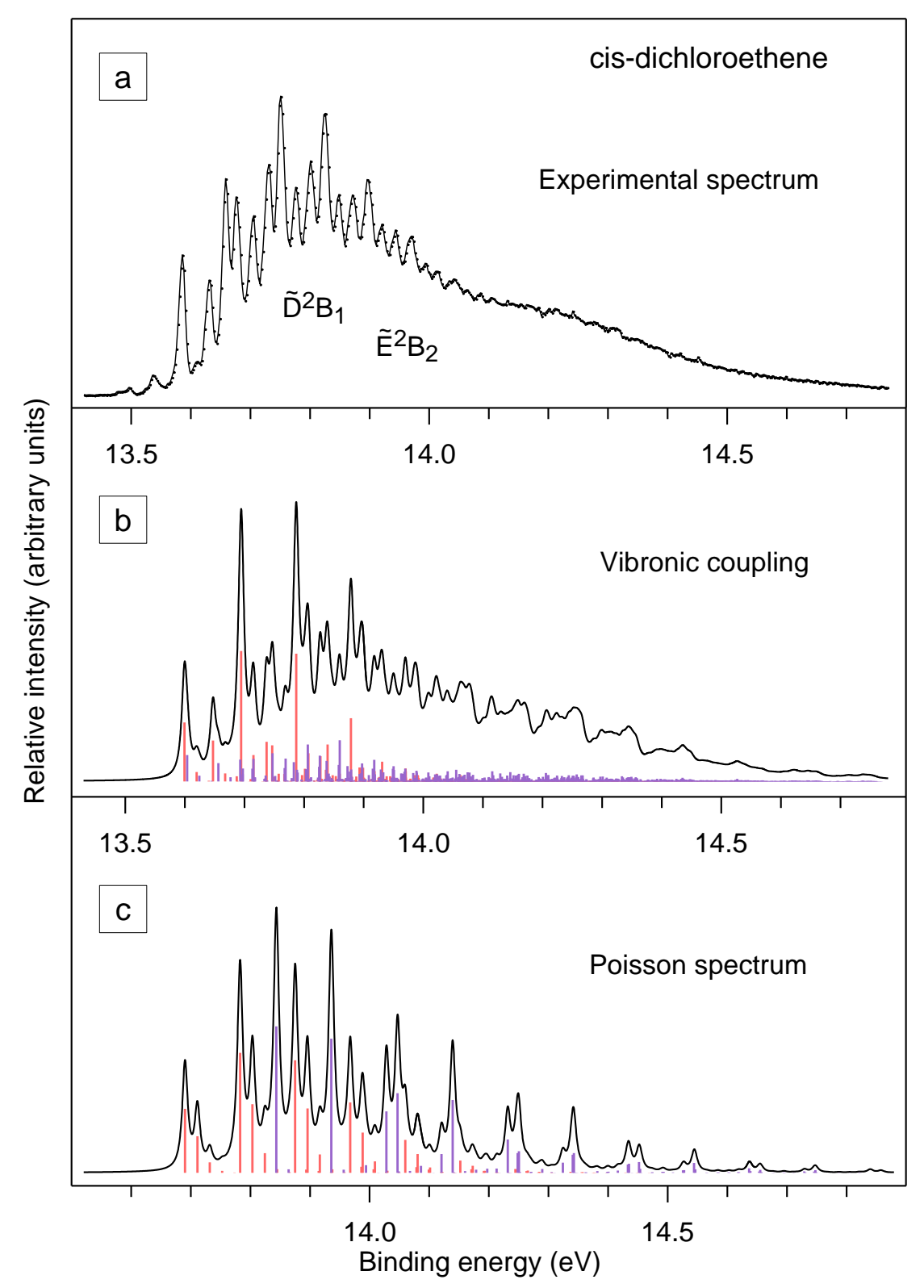

Figure 6 


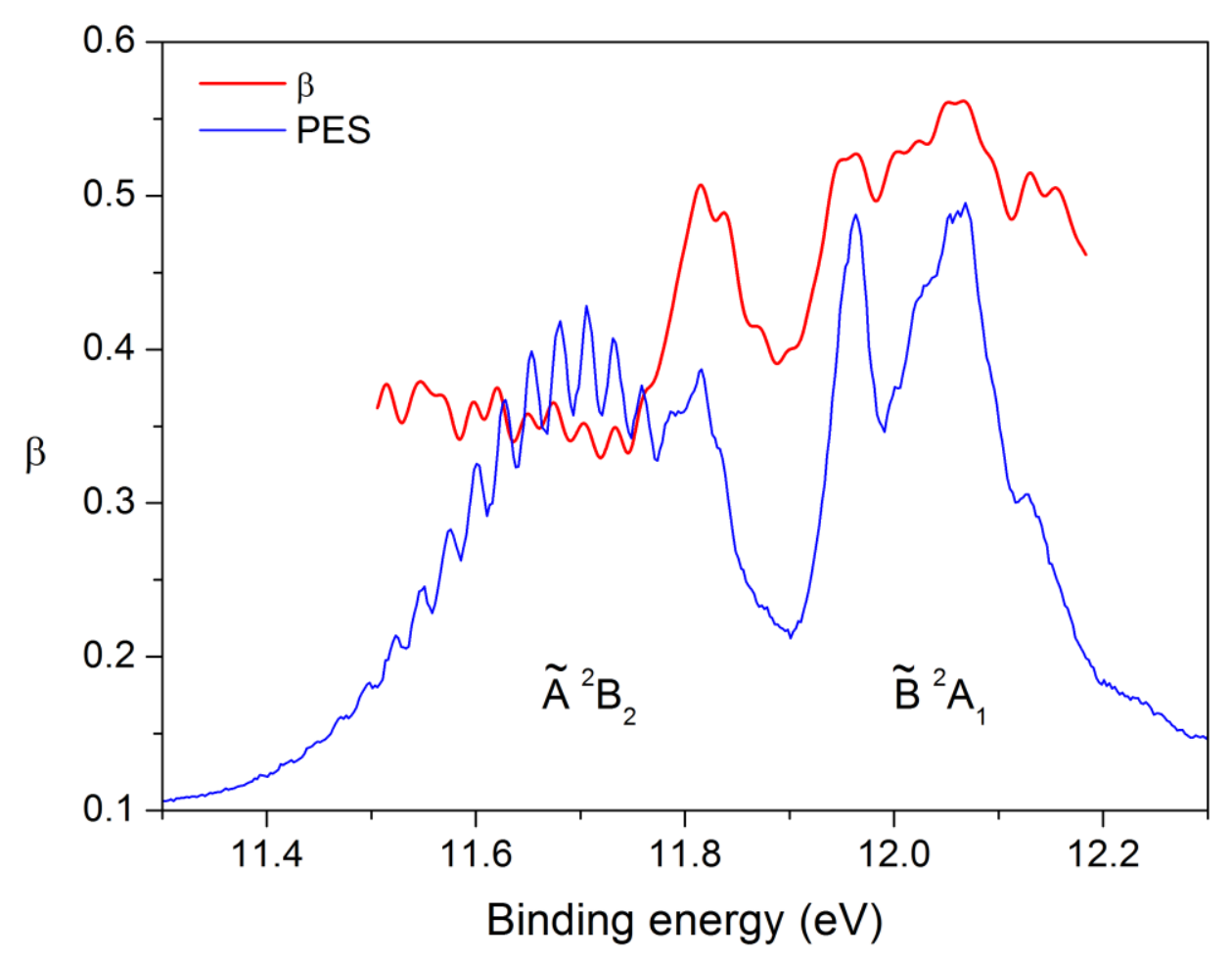

Figure 7 